\title{
Magnetic-field-induced structural phase transition and giant magnetoresistance in $\mathrm{La}_{0.85} \mathrm{Ce}_{0.15} \mathrm{Fe}_{12} \mathrm{~B}_{6}$
}

\author{
L. V. B. Diop $\odot,{ }^{1, *}$ T. Faske, ${ }^{2}$ O. Isnard $\odot,{ }^{3}$ and W. Donner $\odot^{2}$ \\ ${ }^{1}$ Université de Lorraine, CNRS, IJL, F-54000 Nancy, France \\ ${ }^{2}$ Institute of Materials Science, Technical University of Darmstadt, D-64287 Darmstadt, Germany \\ ${ }^{3}$ Université Grenoble Alpes, Institut NÉEL, CNRS, 25 rue des martyrs, F-38042 Grenoble, France
}

(Received 7 June 2021; revised 3 August 2021; accepted 13 September 2021; published 4 October 2021)

\begin{abstract}
Magnetoelastic coupling, structural, magnetic, electronic transport, and magnetotransport properties of $\mathrm{La}_{0.85} \mathrm{Ce}_{0.15} \mathrm{Fe}_{12} \mathrm{~B}_{6}$ have been studied by a combination of macroscopic [magnetization, electrical resistivity, and magnetoresistance (MR)] and microscopic temperature- and magnetic-field-dependent $\mathrm{x}$-ray powder diffraction measurements. The itinerant-electron system $\mathrm{La}_{0.85} \mathrm{Ce}_{0.15} \mathrm{Fe}_{12} \mathrm{~B}_{6}$ exhibits an antiferromagnetic (AFM) ground state and multiple magnetic transitions, AFM-ferromagnetic (FM) and FM-paramagnetic (PM), triggered by changes in both temperature and magnetic fields. At low temperatures, the field-induced first-order AFM-FM metamagnetic phase transition is discontinuous, manifesting itself by extremely sharp steps in magnetization as well as in MR and is accompanied by large magnetic hysteresis. A remarkably large negative MR of $-73 \%$ was discovered. In addition, the time evolution of the electrical resistivity displays a colossal spontaneous jump when both the applied magnetic field and temperature are constant. Diffraction data reveal a magnetic-field-induced structural phase transition associated with the AFM-FM and PM-FM transformations. The lattice distortion is driven by magnetoelastic coupling and converts the crystal structure from rhombohedral $(R \overline{3} m)$ to monoclinic $(C 2 / m)$. The AFM and PM states are related to the rhombohedral structure, whereas the FM order develops in the monoclinic symmetry. A huge volume magnetostriction of $\sim 0.9 \%$ accompanies this symmetry-lowering lattice distortion. Meanwhile, a highly anisotropic thermal expansion involving giant negative thermal expansion with an average volumetric thermal expansion coefficient $\alpha_{V}=-195 \times 10^{-6} \mathrm{~K}^{-1}$ was observed. The consistency seen in these different experimental data constitutes direct evidence of the strong correlations between charge, magnetic, and crystallographic degrees of freedom in this material.
\end{abstract}

DOI: 10.1103/PhysRevMaterials.5.104401

\section{INTRODUCTION}

It is general knowledge that many compounds may undergo structural phase transitions when subject to changes in hydrostatic pressure, temperature, and chemical composition. However, the occurrence of structure transformations induced by applied magnetic field is rather rare, and only a few examples are discussed in the literature. Materials with interconnected lattice and spin degrees of freedom often exhibit multifunctional properties such as giant magnetoresistance (MR), colossal magnetostriction, and giant magnetocaloric effect [1-11]. These prominent magnetoresponsive physical properties of relevance result from instabilities in magnetic and crystallographic sublattices [9]. That is, these emergent physical phenomena are particularly pronounced in the vicinity of a first-order magnetostructural phase transformation, which in turn allows controlling of the physical properties of the solid system via several types of externally applied driving forces. At present, the different families of materials featuring a strong magnetoelastic coupling are of great importance from the fundamental research side as well as from the technological applications viewpoint.

*leopold.diop@univ-lorraine.fr
Understanding the interplay between crystallographic and magnetic sublattices is a crucial challenge in condensed matter science. These magnetic systems form a phenomenal playground for materials physics due to the extreme sensitivity of their physical properties to reasonably weak external stimuli.

Most recently, discontinuous and unconventional staircaselike metamagnetic phase transitions were discovered in the ( $\mathrm{La}, \mathrm{Ce}) \mathrm{Fe}_{12} \mathrm{~B}_{6}$ system [12-15]. This singular multistep behavior is featured by steep magnetization jumps followed by plateaus leading to a unique and unusual avalanchelike magnetization process. The itinerant-electron metamagnet $\mathrm{LaFe}_{12} \mathrm{~B}_{6}$ occupies a special place among rare-earth iron-rich intermetallic compounds; it presents uncommon magnetic behavior and many intriguing physical properties among which the amplitude-modulated antiferromagnetic (AFM) structure described by a propagation vector $\mathbf{k}=\left(\frac{1}{4}, \frac{1}{4}, \frac{1}{4}\right)$, especially weak Fe moment $\left(0.43 \mu_{\mathrm{B}}\right)$ in the magnetically ordered ground state, remarkably low magnetic ordering temperature $T_{\mathrm{N}}=36 \mathrm{~K}$ for an Fe-rich alloy, and a multicritical point in the complex magnetic phase diagram [12]. In addition, both inverse and normal magnetocaloric effects [16], giant spontaneous magnetization steps [14], and large magnetovolume effects [17] can be emphasized as the most relevant intriguing physical properties. These peculiar features not only offer 
the development of experiments under extreme conditions and theoretical models for a better comprehension of the fascinating physics underlying the striking behavior of this compound [17-21], but also highlight the potential interest of the $\mathrm{LaFe}_{12} \mathrm{~B}_{6}$ system for future low-temperature energy technologies. Within the ternary system $R T_{12} \mathrm{~B}_{6}$ (where $R$ is a rare-earth atom, and $T$ stands for a $3 d$ transition metal element $\mathrm{Co}$ or $\mathrm{Fe}$ ), $\mathrm{LaFe}_{12} \mathrm{~B}_{6}$ is the sole stable Fe-based compound of the 1:12:6 family [20,21], whereas the $R \mathrm{Co}_{12} \mathrm{~B}_{6}$ intermetallics are stable along the entire rare-earth series [22]. Even though the $\mathrm{NdFe}_{12} \mathrm{~B}_{6}$ alloy is the first Fe-based member of the $R T_{12} \mathrm{~B}_{6}$ ternary system to be identified, it is metastable [23]. Among the 1:12:6 family, $\mathrm{LaFe}_{12} \mathrm{~B}_{6}$ is the unique compound exhibiting an AFM ground state with an ordering temperature much lower than the Curie point of the Co-based $R \mathrm{Co}_{12} \mathrm{~B}_{6}$ ferromagnets $(R=\mathrm{Y}, \mathrm{La}-\mathrm{Sm})$ or ferrimagnets $(R=\mathrm{Gd}-\mathrm{Tm})$ $T_{C}=134-162 \mathrm{~K}$ [22]. The Néel temperature of $\mathrm{LaFe}_{12} \mathrm{~B}_{6}$ is an order of magnitude smaller than the transition temperature of any rare-earth iron-rich binary alloy. Interestingly, extraordinary electronic transport and magnetotransport properties have been most recently discovered in $R \mathrm{Co}_{12} \mathrm{~B}_{6}$ phases with $R=\mathrm{Y}, \mathrm{Gd}$, and Ho [24].

In this paper, we report direct evidence of a coupled magnetic and structural phase transition stimulated by a magnetic field in the itinerant-electron metamagnet $\mathrm{La}_{0.85} \mathrm{Ce}_{0.15} \mathrm{Fe}_{12} \mathrm{~B}_{6}$ as investigated by means of macroscopic (magnetization, electrical resistivity, and MR) and microscopic (temperature- and magnetic-field-dependent $\mathrm{x}$-ray diffraction) experiments. In addition, we discovered that colossal spontaneous resistivity jumps occur in relaxation measurements, i.e., in experimental conditions where both the applied magnetic field and temperature are kept constant.

\section{EXPERIMENTAL DETAILS}

The $\mathrm{La}_{0.85} \mathrm{Ce}_{0.15} \mathrm{Fe}_{12} \mathrm{~B}_{6}$ compound was synthetized by arc melting the mixture of high-purity constituent elements (better than $99.9 \%$ ) under a protective argon gas atmosphere. To ensure compositional homogeneity, the alloy was arc melted several times with the button being turned over after each remelting. The so-obtained ingot was wrapped in Ta foil, sealed in an evacuated silica tube, and subsequently annealed at $1173 \mathrm{~K}$ for 21 days in a resistive furnace. The analysis of the phase purity and the room temperature crystal structure was performed by standard $\mathrm{x}$-ray powder diffraction using a Siemens D5000 diffractometer in reflection mode with the Bragg-Brentano geometry and $\mathrm{Co}-\mathrm{K} \alpha$ radiation $\left(\lambda_{\mathrm{K} \alpha 1}=\right.$ $1.78897 \AA$ and $\lambda_{\mathrm{K} \alpha 2}=1.79285 \AA$ ).

Magnetic measurements were undertaken on a powder sample using an extraction-type magnetometer. Temperature and magnetic field dependences of the magnetization were measured in applied fields of up to $10.5 \mathrm{~T}$. A detailed description of the magnetometer can be found in Ref. [25]. Magnetization data were corrected for the presence of the minor ferromagnetic $\mathrm{Fe}_{2} \mathrm{~B}$ secondary phase to get the intrinsic magnetic properties of $\mathrm{La}_{0.85} \mathrm{Ce}_{0.15} \mathrm{Fe}_{12} \mathrm{~B}_{6}$. Two different methods were used to determine the amount of $\mathrm{Fe}_{2} \mathrm{~B}$ impurity: (i) X-ray powder diffraction analysis and (ii) magnetization measurements. The latter measurements were realized just above the magnetic ordering temperature of $\mathrm{La}_{0.85} \mathrm{Ce}_{0.15} \mathrm{Fe}_{12} \mathrm{~B}_{6}$ to remain far below the Curie point of $\mathrm{Fe}_{2} \mathrm{~B}$, which is $1015 \mathrm{~K}$. Therefore, the traces of $\mathrm{Fe}_{2} \mathrm{~B}$ impurity were considered as carrying a saturated magnetic moment simplifying the correction for its ferromagnetic contribution. The impurity concentration is estimated to be $\sim 6$ wt. $\%$.

The specimen for the resistivity and MR experiments was cut in parallelepiped form using a diamond saw, and then smooth and flat surfaces were prepared by polishing. The electrical connections on the surface of the sample were made by fixing thin platinum wires using silver paste. The measurements were performed using the conventional four-point contact method at a constant direct current $(\mathrm{dc})$ of $10 \mathrm{~mA}$ at temperatures ranging between 2.5 and $150 \mathrm{~K}$ in a superconducting magnet providing a maximum magnetic field of 8 T. The magnetic field was applied perpendicular to the current orientation $(H \perp i)$. To get rid of possible thermals, the electrical current was applied in opposite polarities at each measurement. The temperature-dependent electrical resistivity curves were recorded at a heating/cooling rate of $1 \mathrm{~K} / \mathrm{min}$. The field dependence of electrical resistivity was measured at a sweep rate of $0.05 \mathrm{~T} / \mathrm{min}$.

$\mathrm{X}$-ray diffraction as a function of both temperature and magnetic field was carried out on a custom-built powder diffractometer in transmission geometry using Mo-K $\alpha$ radiation $\left(\lambda_{\mathrm{K} \alpha 1}=0.70932 \AA\right.$ and $\left.\lambda_{\mathrm{K} \alpha 2}=0.71340 \AA\right)$. Fine powder of $\mathrm{La}_{0.85} \mathrm{Ce}_{0.15} \mathrm{Fe}_{12} \mathrm{~B}_{6}$ was uniformly mixed with a National Institute of Standards and Technology standard reference Si powder $640 \mathrm{~d}$ and then glued onto a carbon foil. The carbon foil was fixed on a copper cold finger-serving as a sample holder-of a closed-cycle helium cryofurnace and transferred into a split-coil superconducting magnet that provided a homogeneous magnetic field of up to $5.5 \mathrm{~T}$ around the sample position with the magnetic field vector perpendicular to the scattering plane. The laboratory-based $\mathrm{x}$-ray powder diffractometer was described in Ref. [26]. Zero-field cooled (ZFC) and field cooled (FC) measuring protocols were employed for thermodiffraction measurements at various applied magnetic fields (isofield measurements). For ZFC mode, the sample was first cooled in zero magnetic field from room temperature down to the lowest measurement temperature. Then the magnetic field was applied after reaching thermal equilibrium, and diffraction data were collected upon heating. The ZFC experimental procedure was immediately followed by cooling under the same applied field (FC). Prior to the magnetic-field-dependent diffraction experiments (isothermal measurements), the sample was cooled from room temperature to the measurement temperature with no magnetic field applied. For both isofield and isothermal experiments, the temperature of the sample was stabilized for $\approx 10 \mathrm{~min}$ before data acquisition. Rietveld refinements of the x-ray powder diffraction patterns were performed using the FULLPROF program [27]. Structural parameters and phase concentrations, when two structurally distinct phases coexisted in certain combinations of magnetic field and temperature, were determined. The sample used in this paper was from the same batch as that employed previously in magnetic relaxation experiments (Ref. [15]). 


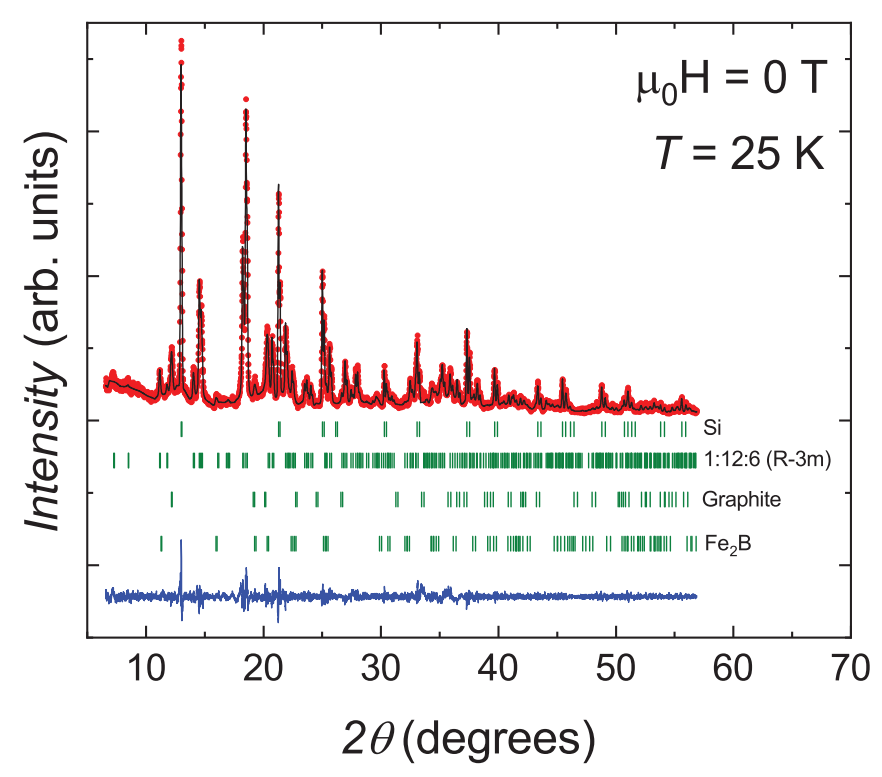

FIG. 1. Rietveld refinement of the $\mathrm{x}$-ray powder diffraction pattern collected at $25 \mathrm{~K}$ in $\mu_{0} \mathrm{H}=0 \mathrm{~T}$ for the $\mathrm{La}_{0.85} \mathrm{Ce}_{0.15} \mathrm{Fe}_{12} \mathrm{~B}_{6}$ compound. The observed (red dots) and calculated (black lines) patterns are shown. The blue line represents the difference $I_{\text {obs }}-I_{\text {calc }}$. The vertical bars (olive) indicate positions of Bragg peaks for the different phases.

\section{RESULTS AND DISCUSSION}

\section{A. X-ray powder diffraction in zero magnetic field $\mu_{0} H=0 \mathrm{~T}$}

When the intermetallic compound $\mathrm{La}_{0.85} \mathrm{Ce}_{0.15} \mathrm{Fe}_{12} \mathrm{~B}_{6}$ is cooled in zero magnetic field $\left(\mu_{0} H=0 \mathrm{~T}\right)$ from room temperature down to $15 \mathrm{~K}$, its $\mathrm{x}$-ray powder diffraction diagrams remain identical except for an anisotropic shift of the Bragg lines toward higher diffraction angles consistent with thermal shrinkage. The Rietveld analyses indicated that, as long as the alloy remains AFM below the Néel temperature or paramagnetic (PM) above, it possesses the rhombohedral $\mathrm{SrNi}_{12} \mathrm{~B}_{6}$-type crystal structure with $R \overline{3} m$ space group [28-30]. The lattice symmetry is preserved over the entire investigated temperature interval, i.e., the trigonal symmetry of the atomic arrangement is kept unchanged. No signature of a temperature-induced structural phase transition was perceived down to $15 \mathrm{~K}$. Within the unit cell, Fe atoms are located on two inequivalent Wyckoff positions, namely, $18 \mathrm{~g}$ and $18 h$. The $\mathrm{B}$ and $\mathrm{La} / \mathrm{Ce}$ atoms reside on the $18 h$ and $3 a$ sites, respectively. A Rietveld refinement of the diffraction pattern recorded at $25 \mathrm{~K}$ in zero magnetic field $\left(\mu_{0} H=0 \mathrm{~T}\right)$ is shown in Fig. 1, and the resulting parameters are summarized in Table I.

The unit cell dimensions $a$ and $c$, volume $V$, and $c / a$ ratio of the rhombohedral structure are plotted in Fig. 2 as a function of temperature. The zero magnetic field thermodiffraction results demonstrate that both lattice parameters are anisotropically decreased as the temperature is lowered, yielding a reduction in the unit cell volume. By contrast, the $c / a$ ratio shows the opposite trend upon cooling. At high temperatures, the structural parameters evolve nearly linearly, and the determined coefficients of linear thermal expansion along the two principal crystallographic directions amount to $\alpha_{a}=$ $13.3 \times 10^{-6} \mathrm{~K}^{-1}$ and $\alpha_{c}=4.9 \times 10^{-6} \mathrm{~K}^{-1}$. The thermal expansion along the high-symmetry direction $c$ is much smaller than that along the $a$ axis (basal plane). The crystallographic volume thermal expansion coefficient is estimated to be about $\alpha_{V}=31.5 \times 10^{-6} \mathrm{~K}^{-1}$. In the temperature range below $60 \mathrm{~K}$, the lattice parameter $a$ remains basically constant, and
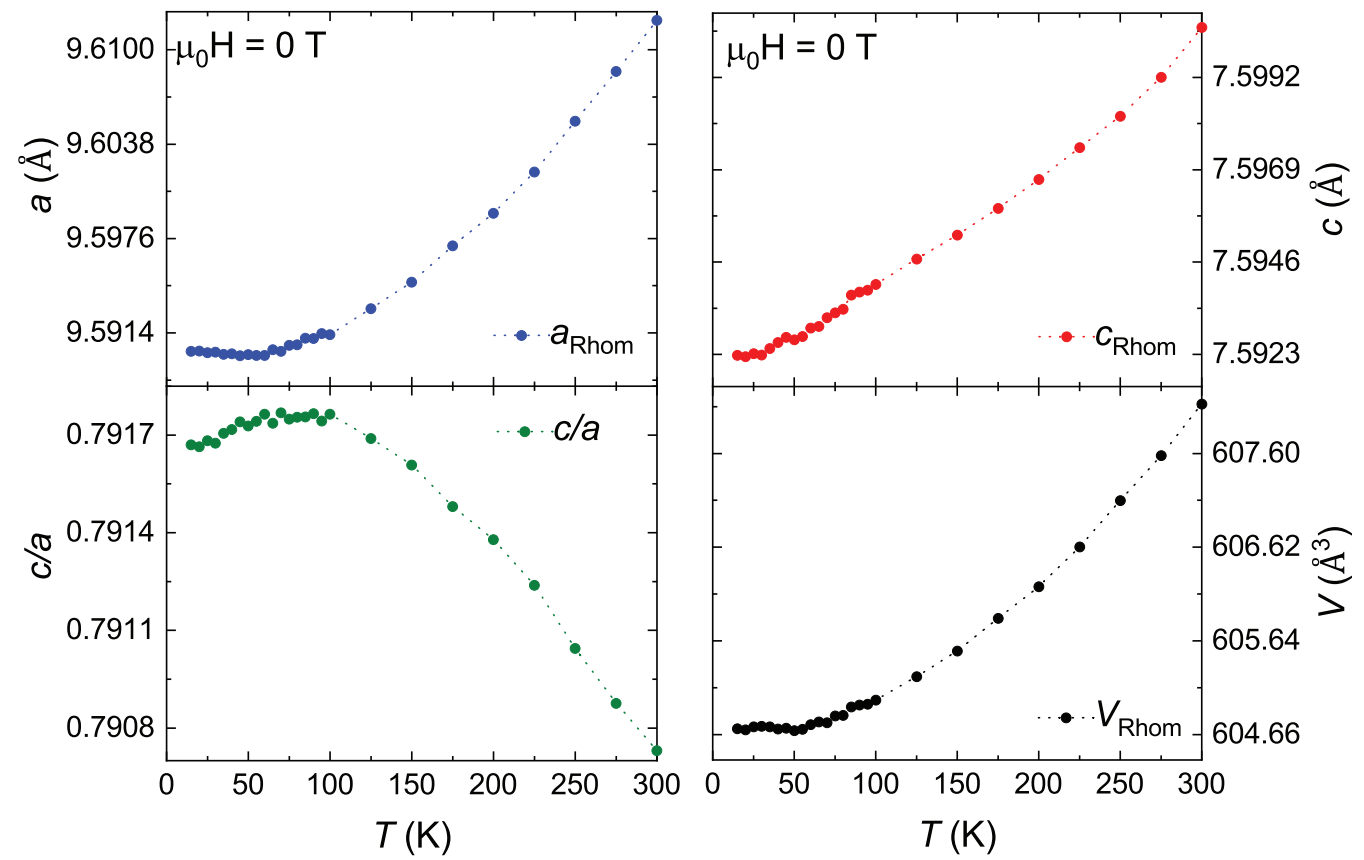

FIG. 2. Temperature dependence of the lattice parameters $a$ and $c, c / a$ ratio, and unit cell volume $V$ of the rhombohedral cell for $\mathrm{La}_{0.85} \mathrm{Ce}_{0.15} \mathrm{Fe}_{12} \mathrm{~B}_{6}$ as derived from Rietveld refinement of the diffraction patterns collected upon cooling in $\mu_{0} H=0 \mathrm{~T}$. Dashed lines are guide for the eye. 
TABLE I. Structural parameters of the $\mathrm{La}_{0.85} \mathrm{Ce}_{0.15} \mathrm{Fe}_{12} \mathrm{~B}_{6}$ compound deduced from Rietveld refinement of the x-ray diffraction patterns collected at $T=25 \mathrm{~K}$ in magnetic field of 0 and $4 \mathrm{~T}$. Crystallographic space group, lattice parameters, unit cell volume, number of chemical formula in unit cell, phase fraction, fractional atomic coordinates, and reliability factors are indicated.

\begin{tabular}{|c|c|c|c|c|c|c|}
\hline & & \multicolumn{2}{|c|}{$25 \mathrm{~K}$} & & & \multirow{2}{*}{$\begin{array}{c}25 \mathrm{~K} \\
4 \mathrm{~T}\end{array}$} \\
\hline & & $0 \mathrm{~T}$ & $4 \mathrm{~T}$ & & & \\
\hline Space group & & $R-3 m$ & $R-3 m$ & & & $C 2 / m$ \\
\hline$a(\AA)$ & & $9.5903(3)$ & $9.5903(1)$ & & & $7.5755(9)$ \\
\hline$b(\AA)$ & & $9.5903(3)$ & $9.5903(1)$ & & & $9.7817(8)$ \\
\hline$c(\AA)$ & & $7.5923(4)$ & $7.5944(2)$ & & & $5.9667(6)$ \\
\hline$\beta\left(^{\circ}\right)$ & & 90 & 90 & & & $113.089(12)$ \\
\hline$V\left(\AA^{3}\right)$ & & $604.74(7)$ & $604.91(22)$ & & & 406.73(8) \\
\hline$Z$ formula unit & & 3 & 3 & & & 2 \\
\hline$W t(\%)$ & & 100 & 19.8 & & & 80.2 \\
\hline \multirow[t]{3}{*}{$\mathrm{La} / \mathrm{Ce}(3 a)$} & $x$ & 0.0000 & 0.0000 & $\mathrm{La} / \mathrm{Ce}(2 c)$ & $x$ & 0.0000 \\
\hline & $y$ & 0.0000 & 0.0000 & & $y$ & 0.0000 \\
\hline & $z$ & 0.0000 & 0.0000 & & $z$ & 0.5000 \\
\hline \multirow[t]{6}{*}{ Fe1 $(18 h)$} & $x$ & $0.4254(6)$ & $0.4245(7)$ & $\mathrm{Fe} 1 A(4 i)$ & $x$ & 0.5407 \\
\hline & $y$ & $-0.4254(6)$ & $-0.4245(7)$ & & $y$ & 0.0000 \\
\hline & $z$ & $0.0366(5)$ & $0.0331(8)$ & & $z$ & 0.3121 \\
\hline & & & & $\mathrm{Fe} 1 B(8 j)$ & $x$ & 0.1764 \\
\hline & & & & & $y$ & 0.3643 \\
\hline & & & & & $z$ & 0.0407 \\
\hline \multirow[t]{6}{*}{$\mathrm{Fe} 2(18 g)$} & $x$ & $0.3698(3)$ & $0.3691(5)$ & $\mathrm{Fe} 2 A(8 j)$ & $x$ & 0.3158 \\
\hline & $y$ & 0.0000 & 0.0000 & & $y$ & 0.1842 \\
\hline & $z$ & 0.5000 & 0.5000 & & $z$ & 0.3684 \\
\hline & & & & $\mathrm{Fe} 2 B(4 g)$ & $x$ & 0.0000 \\
\hline & & & & & $y$ & 0.1316 \\
\hline & & & & & $z$ & 0.0000 \\
\hline \multirow[t]{6}{*}{ B1 (18h) } & $x$ & $0.1631(5)$ & $0.1578(7)$ & $\mathrm{B} 1 A(4 i)$ & $x$ & 0.2333 \\
\hline & $y$ & $-0.1631(5)$ & $-0.1578(7)$ & & $y$ & 0.0000 \\
\hline & $z$ & $0.0659(6)$ & $0.0772(9)$ & & $z$ & 0.1598 \\
\hline & & & & $\mathrm{B} 1 B(8 j)$ & $x$ & 0.0535 \\
\hline & & & & & $y$ & 0.2868 \\
\hline & & & & & $z$ & 0.2667 \\
\hline$\chi^{2}$ & & 1.62 & 1.54 & & & 1.54 \\
\hline$R_{\text {Bragg }}(\%)$ & & 4.88 & 4.97 & & & 4.72 \\
\hline$R_{\mathrm{wp}}(\%)$ & & 9.03 & 8.63 & & & 8.63 \\
\hline$R_{\mathrm{p}}(\%)$ & & 7.12 & 6.78 & & & 6.78 \\
\hline$R_{\exp }(\%)$ & & 7.08 & 6.96 & & & 6.96 \\
\hline
\end{tabular}

consequently, the thermal expansion along this crystallographic direction approaches zero. On the other hand, the thermal expansion along the threefold symmetry axis $c$ becomes almost negligible only below $30 \mathrm{~K}$. This difference on the temperature at which a practically zero thermal expansion is observed along the two principal crystallographic axes is explainable by considering the anisotropy of the lattice. The flattening of the linear thermal expansion at low temperatures is in accord with the normal phonon contribution approaching zero (in agreement with Grüneisen's law). In the thermal variation of the volume, no discernible abnormality is detected at $T_{\mathrm{N}}$ associated with the second-order AFM-PM transition.

\section{B. Magnetization}

Figure 3 displays the temperature-dependent magnetization curves $M(T)$ measured under various applied magnetic fields between 0.1 and $9.5 \mathrm{~T}$. The low-field thermomagnetic curve [Fig. 3(a)] presents a small peak around $T_{\mathrm{N}}=35 \mathrm{~K}$, indicating that $\mathrm{La}_{0.85} \mathrm{Ce}_{0.15} \mathrm{Fe}_{12} \mathrm{~B}_{6}$ undergoes a second-order transition from an AFM to a PM phase. The $M(T)$ measurements recorded in an applied field of $9.5 \mathrm{~T}$ [Fig. 3(b)] reflect the magnetic transition from a typical FM phase to a PM state. The $4 \mathrm{~T}(6 \mathrm{~T})$ thermomagnetic curve reveals that a large percentage $\sim 74 \%$ (80\%) of the sample volume is transformed into a FM phase at low temperatures, and the remaining fraction $\sim 26 \%$ (20\%) is in the AFM ground state. Note that the concentration of induced FM phase is strongly dependent on the strength of applied field, which favors the FM order over the AFM phase.

Nevertheless, the magnetization presents considerably different temperature dependence when conducted in $2 \mathrm{~T}$ magnetic field. The ZFC $M(T)$ curve $\left(\mu_{0} H=2 \mathrm{~T}\right)$ manifests a bell-shaped anomaly, and two magnetic events occur sequentially upon heating. The first one corresponds to an AFM-to-FM phase transition at low temperatures, and the 

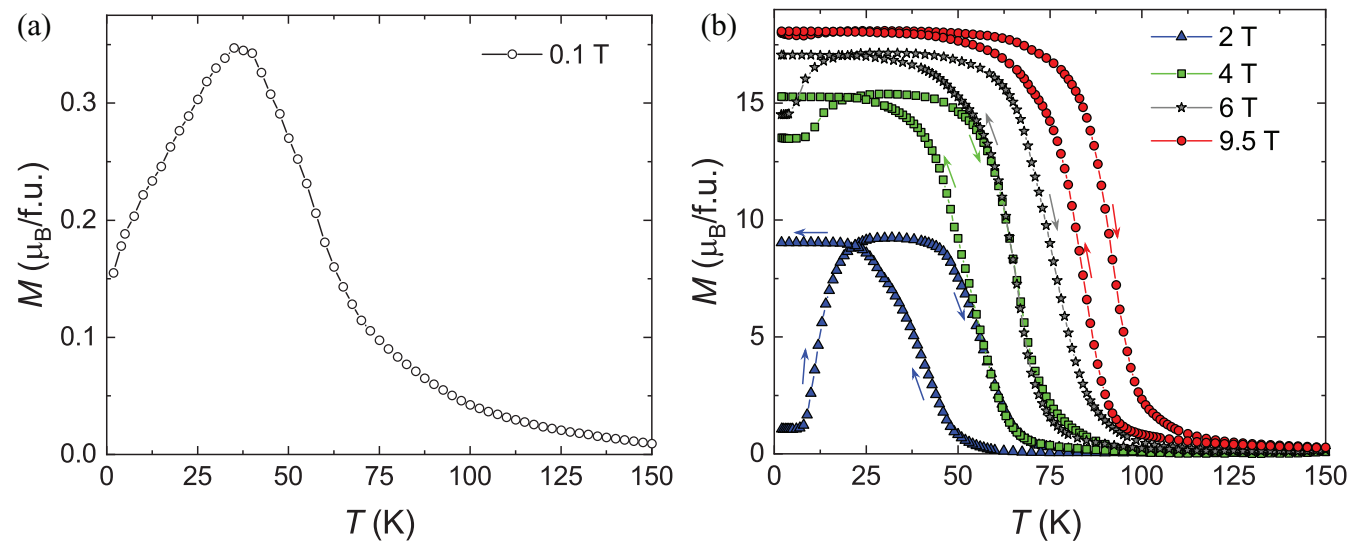

FIG. 3. Temperature dependence of the magnetization of $\mathrm{La}_{0.85} \mathrm{Ce}_{0.15} \mathrm{Fe}_{12} \mathrm{~B}_{6}$ : (a) magnetization measured using zero-field cooled (ZFC) protocol in $0.1 \mathrm{~T}$ applied magnetic field; (b) magnetization measured in applied magnetic fields of 2, 4, 6, and 9.5 T [both ZFC and field cooled (FC) data are marked by the same symbols. The arrows indicate the direction of the temperature change].

second one is a FM-to-PM transformation at high temperatures. The spectacular increase in the magnetization by $550 \%$, when temperature is raised by $8 \mathrm{~K}$, is associated with the sudden formation of the FM order. Throughout this magnetic phase transition, both AFM and FM ordered states coexist, hence forming a magnetically heterogeneous state (magnetic-phase-segregated state). Cooling in a $2 \mathrm{~T}$ magnetic field converts $\mathrm{La}_{0.85} \mathrm{Ce}_{0.15} \mathrm{Fe}_{12} \mathrm{~B}_{6}$ into a partially $\mathrm{FM}$ state. The $2 \mathrm{~T}$ curve shows a pronounced splitting between ZFC and FC modes, and the maximum value of magnetization for ZFC measuring protocol is larger than for the FC branch. This last aspect is rather unusual for standard ferromagnetic systems in an applied magnetic field as large as 2 T. A similar bell-like anomaly was evidenced in the parent compound $\mathrm{LaFe}_{12} \mathrm{~B}_{6}$ within the magnetic field range between 4.75 and $7 \mathrm{~T}$ [12]. Another salient feature of the isofield magnetization curves of $\mathrm{La}_{0.85} \mathrm{Ce}_{0.15} \mathrm{Fe}_{12} \mathrm{~B}_{6}$ is the huge temperature hysteresis of $\sim 12 \mathrm{~K}$ in the vicinity of the FM-PM magnetic phase transition, which is consistent with the first-order character of the transformation. Upon increasing the applied magnetic field, the Curie temperature $T_{\mathrm{C}}$ is strongly shifted to higher temperatures.

To elucidate the magnetic states at different fixed temperatures and applied fields, isothermal magnetization curves $M(H)$ were taken on the thermally demagnetized $\mathrm{La}_{0.85} \mathrm{Ce}_{0.15} \mathrm{Fe}_{12} \mathrm{~B}_{6}$. Just the magnetization isotherms at some representative temperatures are displayed in Fig. 4, but all the recorded data were used to construct the phase diagram depicted in Fig. 5. At $2 \mathrm{~K}$, the virgin curve (first magnetization curve) exhibits three ultrasharp jumps followed by plateaus; generating an avalanchelike metamagnetic process like that observed in $\mathrm{LaFe}_{12} \mathrm{~B}_{6}$. These steplike transitions result from conversion of a fraction of the sample from the AFM state into the FM state. The first and second magnetization plateaus correspond to a mixed phase AFM + FM, i.e., a magnetically heterogenous state. The saturation magnetization of the fully FM polarized state amounts to $17.9 \mu_{\mathrm{B}} / \mathrm{f}$.u. No abrupt steps nor transitions are seen in the demagnetization curve, which shows a conventional ferromagnetic behavior, soft magnetism like with no remanent magnetization, and no significant coercivity. After the applied field is reduced to zero, $100 \%$ of the sample remains in the forced FM state, indicating that the AFM-FM phase transformation is completely irreversible at this temperature. The sharpness of the staircaselike transitions decreases with increasing temperature and vanishes at $8 \mathrm{~K}$ where the magnetization process becomes smooth. In $\mathrm{La}_{0.85} \mathrm{Ce}_{0.15} \mathrm{Fe}_{12} \mathrm{~B}_{6}$, the magnetic-field-induced metamagnetic transition extends over a wide temperature interval, below and well above the Néel temperature, and proceeds beyond $8 \mathrm{~K}$ through a progressive conversion of the PM and AFM phases into FM domains upon increasing applied field. The field-driven AFM-FM and PM-FM phase transformations are accompanied by a large magnetic hysteresis, bearing witness to the first-order nature of the metamagnetic transition.

The critical magnetic fields were defined as the peak of the derivative of magnetization with respect to the magnetic field, and the obtained values were used to elaborate the magnetic field $\left(\mu_{0} H\right)$-temperature $(T)$ phase diagram shown in Fig. 5. The critical field of the demagnetization path

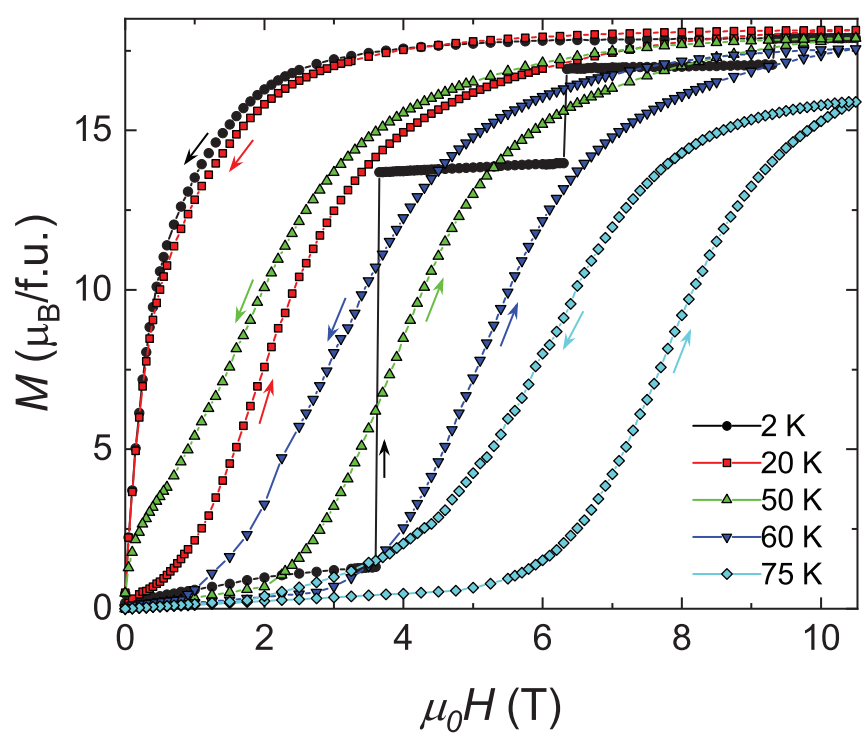

FIG. 4. Magnetization isotherms of $\mathrm{La}_{0.85} \mathrm{Ce}_{0.15} \mathrm{Fe}_{12} \mathrm{~B}_{6}$ measured between 2 and $75 \mathrm{~K}$. 


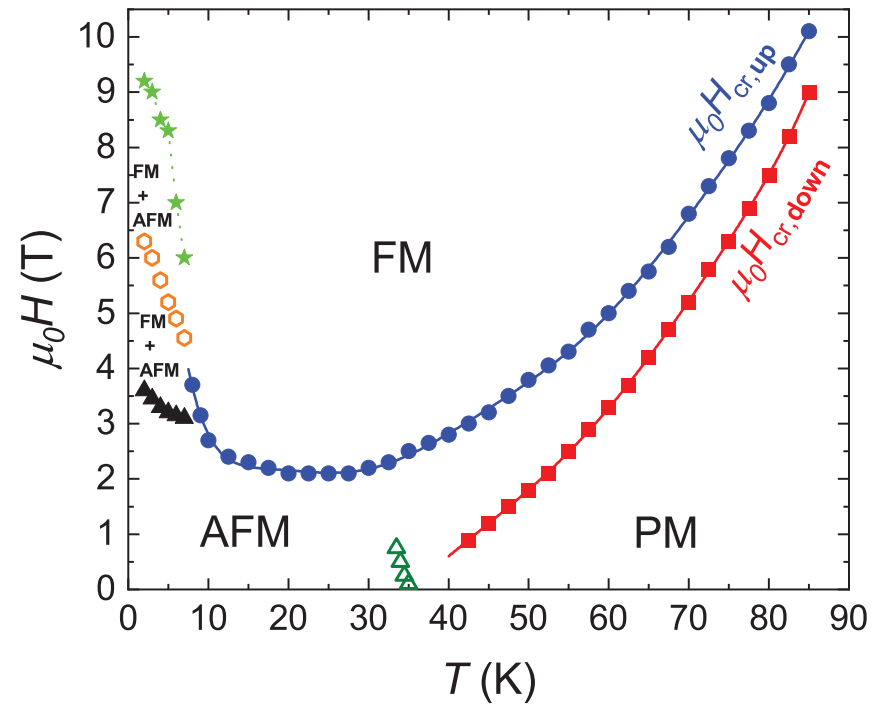

FIG. 5. Magnetic phase diagram of $\mathrm{La}_{0.85} \mathrm{Ce}_{0.15} \mathrm{Fe}_{12} \mathrm{~B}_{6}$. The critical transition field for the upward $\left(\mu_{0} H_{\text {cr,up }}\right)$ and downward $\left(\mu_{0} H_{\text {cr,down }}\right)$ field scans as a function of temperature. The transition magnetic field has been derived from the maximum of the field derivative of the magnetization isotherms. The closed triangles, opened hexagons, and closed stars correspond to the critical field of the first, second, and third jump, respectively, observed on the isothermal magnetization curves. The open triangles represent the Néel temperature $T_{\mathrm{N}}$.

$\mu_{0} H_{\mathrm{cr}, \text { down }}$ varies continuously with temperature. By contrast, the thermal evolution of the critical field obtained for the field-increasing leg $\mu_{0} H_{\text {cr, up }}$ is nonmonotonic. Above $27.5 \mathrm{~K}, \mu_{0} H_{\mathrm{cr}, \text { up }}$ increases upon heating, while it presents the opposite trend at lower temperatures. Below $27.5 \mathrm{~K}$, the transition field of the first-order AFM-FM phase transition rises with lowering the temperature due to the strengthening of the negative exchange interactions and the diminution of the thermal fluctuations of the magnetic moments and elasticity of the crystal lattice in the AFM state [12,15,31]. This leads to the increase of both the free energy difference between the two magnetically ordered AFM and FM phases and the critical field needed to accomplish the magnetic transformation from one state to another. In the PM

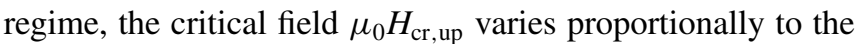
square of temperature $T^{2}$ ( $T^{2}$ dependence) at low temperatures and exhibits a $T$ dependence at high temperatures, in accord with the spin fluctuation theory for itinerant-electron metamagnetic systems [32-34]. The Néel temperature slightly decreases with increasing applied magnetic field. In addition to the three relatively well-delineated magnetic regionsAFM, FM, and PM-another intriguing characteristic of this complex magnetic phase diagram is the low-temperature regime where the itinerant-electron metamagnetic compound $\mathrm{La}_{0.85} \mathrm{Ce}_{0.15} \mathrm{Fe}_{12} \mathrm{~B}_{6}$ may be in an AFM, FM, or magnetically heterogeneous AFM + FM state depending on thermomagnetic history effects. For instance, when $\mathrm{La}_{0.85} \mathrm{Ce}_{0.15} \mathrm{Fe}_{12} \mathrm{~B}_{6}$ is cooled in zero magnetic field from the PM region down to $2 \mathrm{~K}$ and subsequently a field of $3 \mathrm{~T}$ is applied, the sample will stay in the pure AFM ground state at $2 \mathrm{~K}$ and $3 \mathrm{~T}$. When cooled from room temperature down to $2 \mathrm{~K}$ in an applied magnetic field of $3 \mathrm{~T}$, the system will cross the AFM-FM phase boundary and endure a partial AFM $\rightarrow$ FM magnetic phase transformation; in such experimental conditions, the material will stay in the mixed phase (or phase separated) $\mathrm{AFM}+\mathrm{FM}$ state.

\section{Resistivity and MR}

The temperature-dependent electrical resistivity curves $\rho(T)$ recorded during warming and cooling between 2.5 and $150 \mathrm{~K}$ under various magnetic fields are plotted in Fig. 6(a). For each isofield $\rho(T)$ curve, the sample was first slowly cooled to $2.5 \mathrm{~K}$ in zero field. At this temperature of $2.5 \mathrm{~K}$, the desired magnetic field was applied, and resistivity data were collected as the system is heated up to $150 \mathrm{~K}$. Subsequently, the resistivity was measured by cooling the sample from 150 to $2.5 \mathrm{~K}$ under the same constant external field. All $\rho(T)$ plots display a strongly linear increase in resistivity at high temperatures, which is indicative of the metallic character, reflecting the dominance of the electron-phonon contribution. Such linear behavior is the general trend in the PM region, whatever magnetic field strength is used here. In zero magnetic field, both electrical resistivity functions, heating and cooling, of $\mathrm{La}_{0.85} \mathrm{Ce}_{0.15} \mathrm{Fe}_{12} \mathrm{~B}_{6}$ are practically identical, demonstrating that the mechanisms responsible for charge-carrier scattering and their concentration are not affected by the direction of the temperature change. A small anomaly is found at $\approx 33 \mathrm{~K}$ in the $0 \mathrm{~T} \rho(T)$ curves. In magnetic field of $8 \mathrm{~T}(6 \mathrm{~T})$, the dramatic increase in the resistivity at $85 \mathrm{~K}(77 \mathrm{~K})$ upon heating arises from transition between the FM (low resistivity) and PM (high resistivity) phases. These transition temperature values are in excellent agreement with the Curie points derived from thermomagnetic measurements. The increase of external applied field lowers the resistivity around the magnetic ordering temperature $T_{\mathrm{C}}$ because the spin scattering is decreased by the magnetic-field-induced orientation of the local magnetic moments. The large anomaly in electrical resistivity in the vicinity of $T_{\mathrm{C}}$ indicates a strong interaction of Fe magnetic moments with conduction electrons. It is worth recalling that a similar drop of the resistivity has been reported by Mesquita et al. [24] on ferromagnetic $R \mathrm{Co}_{12} \mathrm{~B}_{6}$ isotype compounds.

As it can be clearly seen from Fig. 6(a), the behavior of the electrical resistivity measured in 1.5 and $3.5 \mathrm{~T}$ differs from that observed in zero and high magnetic fields. The $\rho(T)$ curves $\left(\mu_{0} H=1.5\right.$ and $\left.3.5 \mathrm{~T}\right)$ show a strong divergence between $\mathrm{ZFC}$ and FC data and present an even more interesting thermal evolution: on heating from the AFM ground state at $2.5 \mathrm{~K}$, the resistivity exhibits a rapid reduction followed by a plateau, and later, it increases at high temperatures. This peculiar change in resistivity correlates with the presence of both high-temperature FM-PM and low-temperature AFMFM transitions. The onset of FM ordering is featured by a large drop in resistivity. The thermal hysteresis accompanying the FM-PM transformation is remarkably large $\approx 15 \mathrm{~K}$ $\left(\mu_{0} H=1.5 \mathrm{~T}\right)$ and emphasizes the first-order nature of the phase transition. The pronounced change in resistivity across the order-order AFM $\rightarrow$ FM magnetic transformation can be ascribed to the difference in the strength of the scattering of the conduction electrons by the AFM and FM magnons and by the phonons. Our experimental results denote that the 

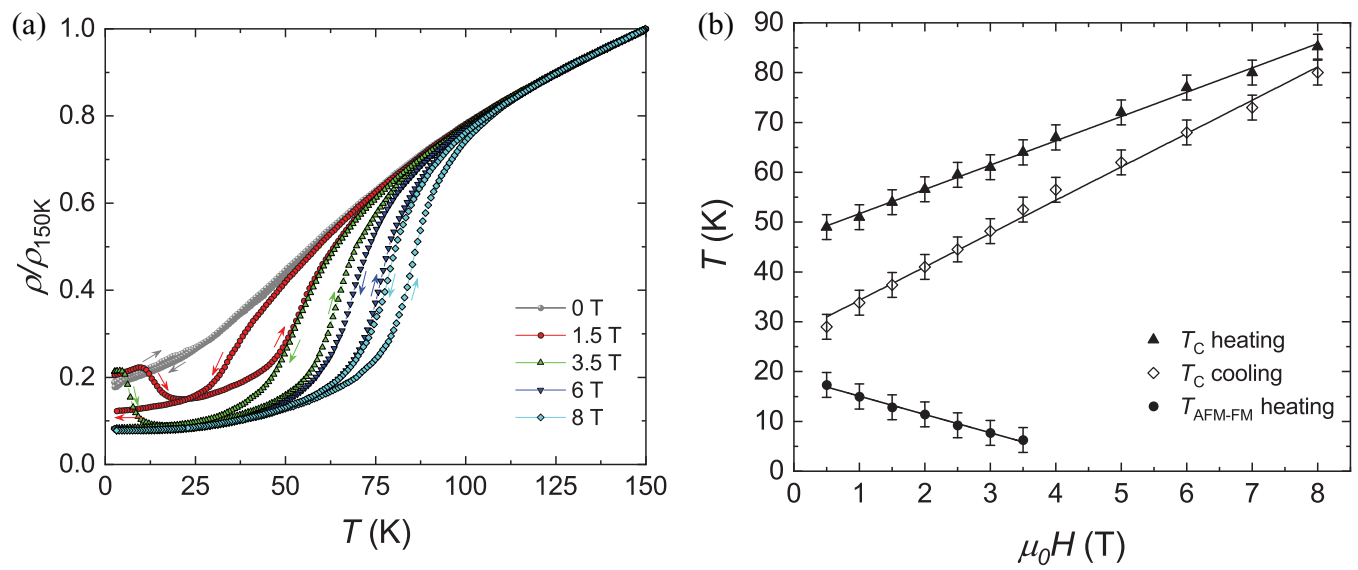

FIG. 6. (a) Temperature dependence of the relative electrical resistivity of $\mathrm{La}_{0.85} \mathrm{Ce}_{0.15} \mathrm{Fe}_{12} \mathrm{~B}_{6}$ on heating and cooling in various applied magnetic fields. Both zero-field cooled (ZFC) and field cooled (FC) data are marked by the same symbols. The arrows indicate the direction of the temperature change. (b) Magnetic field dependence of the transition temperatures.

scattering in the FM structure is smaller than that in the AFM spin configuration because of a larger magnetic order.

The magnetic ordering temperatures $T_{\mathrm{C}}$ and $T_{\mathrm{AFM}-\mathrm{FM}}$ of $\mathrm{La}_{0.85} \mathrm{Ce}_{0.15} \mathrm{Fe}_{12} \mathrm{~B}_{6}$ are plotted in Fig. 6(b) as a function of the applied external field. The application of magnetic field leads to a nearly linear increase of $T_{\mathrm{C}}$ at a rate of 4.9 and $6.7 \mathrm{~K} / \mathrm{T}$ upon heating and cooling, respectively. The other characteristic features of Fig. 6(b) are large thermal hysteresis associated with the transformations and a strong negative shift of $T_{\mathrm{AFM}-\mathrm{FM}}$ with applied magnetic field. These results demonstrate that the magnetic field enhances the FM state.

To probe the correlations between the magnetic degrees of freedom and charge carriers, the magnetic field dependence of the resistivity was measured at various fixed temperatures. Using these data, the MR was assessed as $\left[\rho\left(\mu_{0} H, T\right)-\right.$ $\rho(0, T)] / \rho(0, T)$. Isothermal MR curves are plotted in Figs. 7 and 8 at representative temperature intervals. Each isotherm begins from the virgin state after cooling the sample in zero

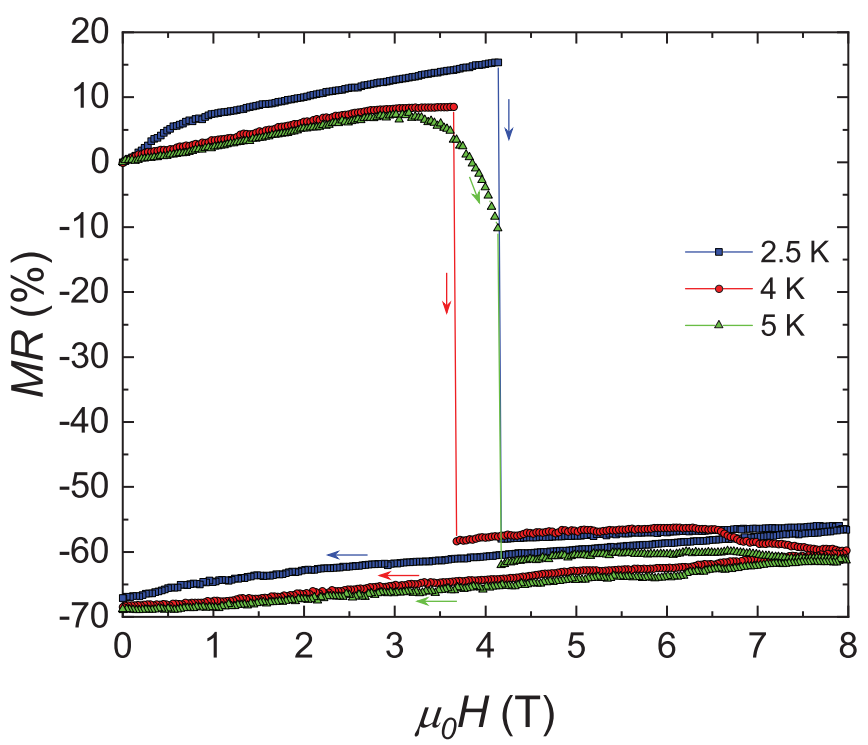

FIG. 7. Magnetic field dependence of the isothermal magnetoresistance of $\mathrm{La}_{0.85} \mathrm{Ce}_{0.15} \mathrm{Fe}_{12} \mathrm{~B}_{6}$ measured at 2.5, 4 and $5 \mathrm{~K}$. field from the PM state. As follows from Fig. 7, during the first field increase, the electrical resistivity initially increases and then exhibits a sharp discontinuity in the same magnetic field range where a pronounced stepwise behavior was observed in the magnetic data. This abrupt and substantial

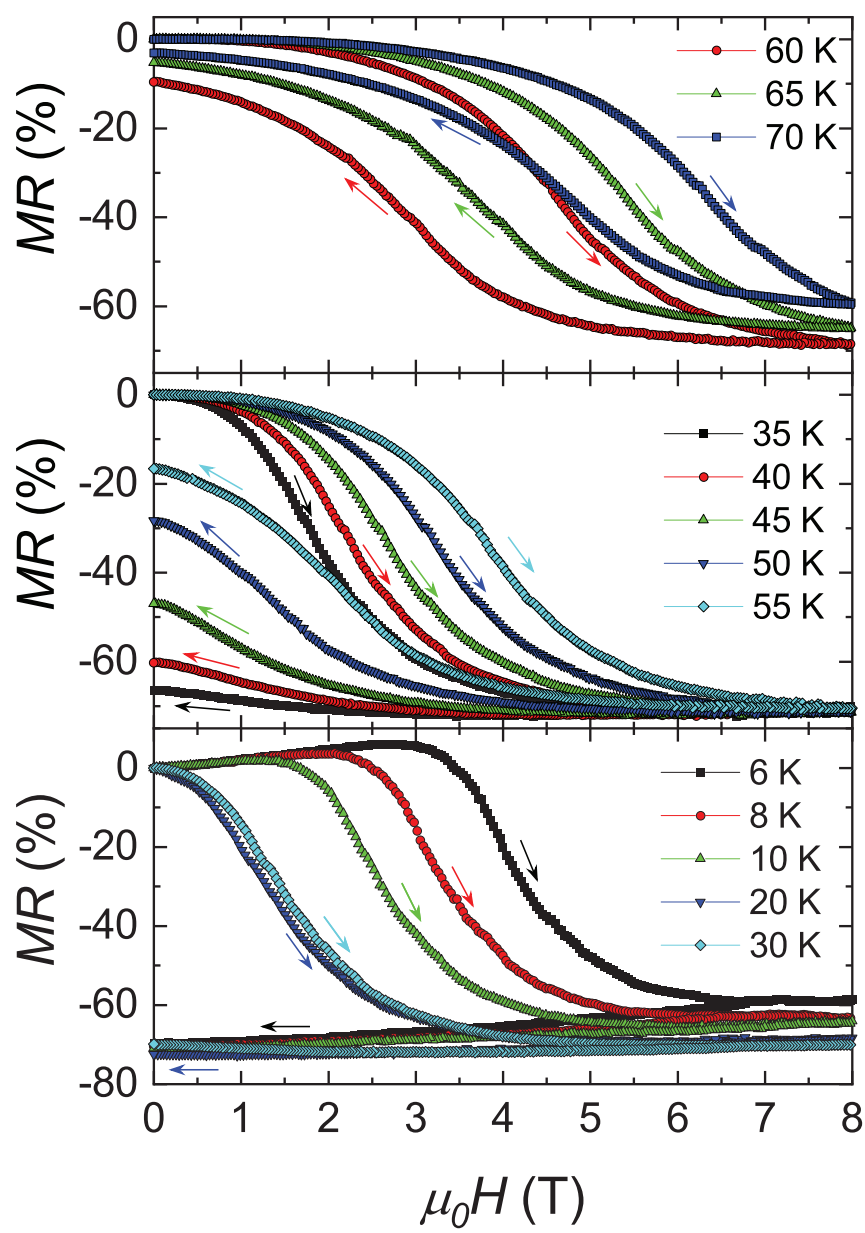

FIG. 8. Magnetoresistance isotherms of $\mathrm{La}_{0.85} \mathrm{Ce}_{0.15} \mathrm{Fe}_{12} \mathrm{~B}_{6}$ at the indicated temperature ranging from 6 to $30 \mathrm{~K}$ (bottom panel), 35 to $55 \mathrm{~K}$ (middle panel) and 60 to $70 \mathrm{~K}$ (top panel). 
change in the resistivity corresponds to a transformation of $\mathrm{La}_{0.85} \mathrm{Ce}_{0.15} \mathrm{Fe}_{12} \mathrm{~B}_{6}$ from an AFM into a FM phase, as proven by the magnetization measurements. The resistivity is larger in the AFM phase when compared with that in the field-driven FM state. The subsequent decreasing field process shows no anomalies because the system stays in the forced FM state, as mentioned above. The compound does not recover the initial value of the resistivity, and a remanent (nonzero) MR appears after removal of the external field. The original AFM ground state and, thus, the initial resistivity value can be restored only after warming the sample above the FM ordering temperature and subsequently cooling without an applied magnetic field. The behavior of the electrical resistivity of $\mathrm{La}_{0.85} \mathrm{Ce}_{0.15} \mathrm{Fe}_{12} \mathrm{~B}_{6}$ supports the conclusion that the magnetic-field-induced phase transition is totally irreversible at very low temperature.

At higher temperatures, the resistivity shows gradual changes across both AFM-FM and PM-FM transformations, unlike the steep jumps observed below $6 \mathrm{~K}$. The MR isotherms display large hysteresis against the magnetic field scan, which is one of the signatures of a first-order transition. Moreover, the hysteretic character and the irreversible/reversible behavior are strongly dependent on the temperature range. We exemplify in Fig. 8 the isothermal MR plots in three different representative temperature intervals: between 6 and $30 \mathrm{~K}$ (bottom panel), from 35 to 55 $\mathrm{K}$ (middle panel), and $T \geqslant 60 \mathrm{~K}$ (top panel). In the AFM phase, below $35 \mathrm{~K}$, the MR ratio is small at low magnetic fields but strikingly decreases above the critical field as the system undergoes a phase transition to the FM state, and a giant negative MR effect is observed. The MR associated with the field-induced first-order AFM-FM metamagnetic phase transformation is estimated to be MR $=-73 \%$ at $20 \mathrm{~K}$. No transition is detected in the decreasing field scan, and the electrical resistivity remains nearly constant down to the zero magnetic field point, clearly pointing out the irreversible nature of the magnetic transition below $35 \mathrm{~K}$. In the temperature range from 35 to $55 \mathrm{~K}$, the decreasing field curve deviates from the pure FM character, and a metamagneticlike transition takes place at lower field, giving rise to a clear hysteresis between both magnetic field processes. From $\sim 35$ to $\sim 55 \mathrm{~K}$, the PM-FM phase transition is partially reversible; a fraction of the sample recovers the PM state when the applied field is reduced to zero. In other words, both irreversible and reversible magnetic transformations exist in this temperature interval. Beyond $60 \mathrm{~K}$, the magnetic-field-induced metamagnetic transformation becomes completely reversible but accompanied with a hysteresis.

As discussed in our previous paper [15], field cooling alters the relative fraction of the different magnetic phases-AFM, FM, and PM. By analogy to the magnetic data, we investigated the influence of the magnetic field strength applied during cooling on the electrical resistivity. For these experiments, the sample was cooled in magnetic field $\left(\mu_{0} H>0\right)$ from high temperatures (PM region) down to the measurement temperature. After stabilizing the desired temperature, the cooling field was removed, and then the resistivity was recorded subsequently as a function of magnetic field up to $8 \mathrm{~T}$ and back to zero field. The corresponding MR results are illustrated in Fig. 9 for some selected temperatures of 2.5, 8, and $45 \mathrm{~K}$ after various field cooling procedures (between 0 and $8 \mathrm{~T}$ ). At

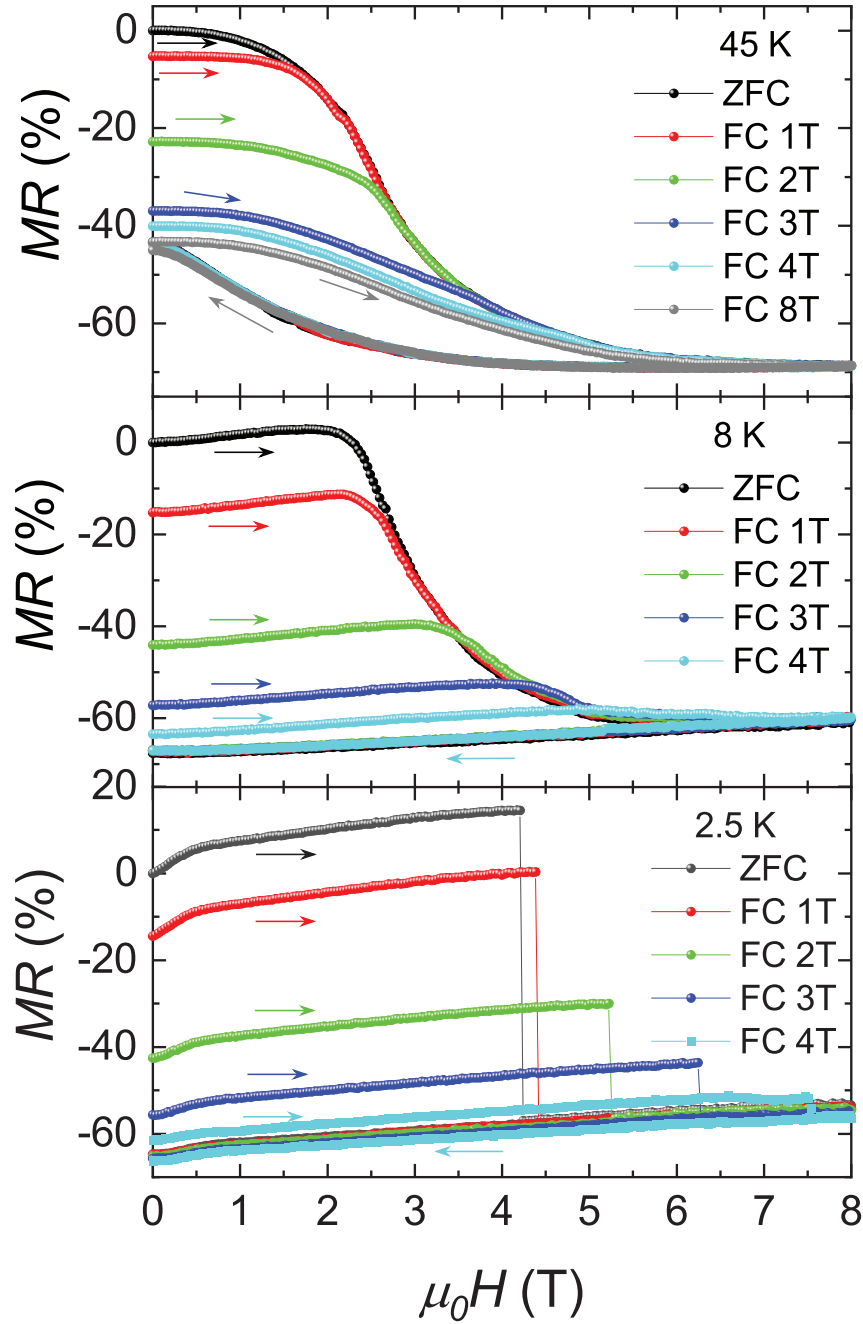

FIG. 9. Isothermal magnetoresistance of $\mathrm{La}_{0.85} \mathrm{Ce}_{0.15} \mathrm{Fe}_{12} \mathrm{~B}_{6}$ at some selected temperatures of $2.5 \mathrm{~K}$ (bottom panel), $8 \mathrm{~K}$ (middle panel), and $45 \mathrm{~K}$ (top panel) taken after cooling the sample in different magnetic fields.

$2.5 \mathrm{~K}$, such a magnetic field cooling process reduces the lowfield resistivity due to the increase of the FM concentration in the sample at the expense of the AFM component. The critical field at which the step transition occurs is fully controlled by the FM phase content. Field cooling shifts the resistivity jump to higher magnetic fields. Cooling in an adequately high external field transforms the system into a fully FM polarized state, suppressing the sharp step. At $8 \mathrm{~K}(45 \mathrm{~K})$, the transition field of the AFM-FM (PM-FM) transformation is hardly changed upon field cooling; however, the thermomagnetic history of the sample strongly affects the fraction of the FM phase and the low-field MR.

Considering the metastability of the different magnetic phases and the anomalous features observed in the data reported above, we further probe time-dependent phenomena (relaxation effect) to study more directly the dynamics of the resistivity abrupt change. The resistive relaxation measurements have been undertaken at $2.5 \mathrm{~K}$ and in magnetic fields slightly below and above the transition field corresponding to the steep jump seen in the MR isotherm. Prior to these time 


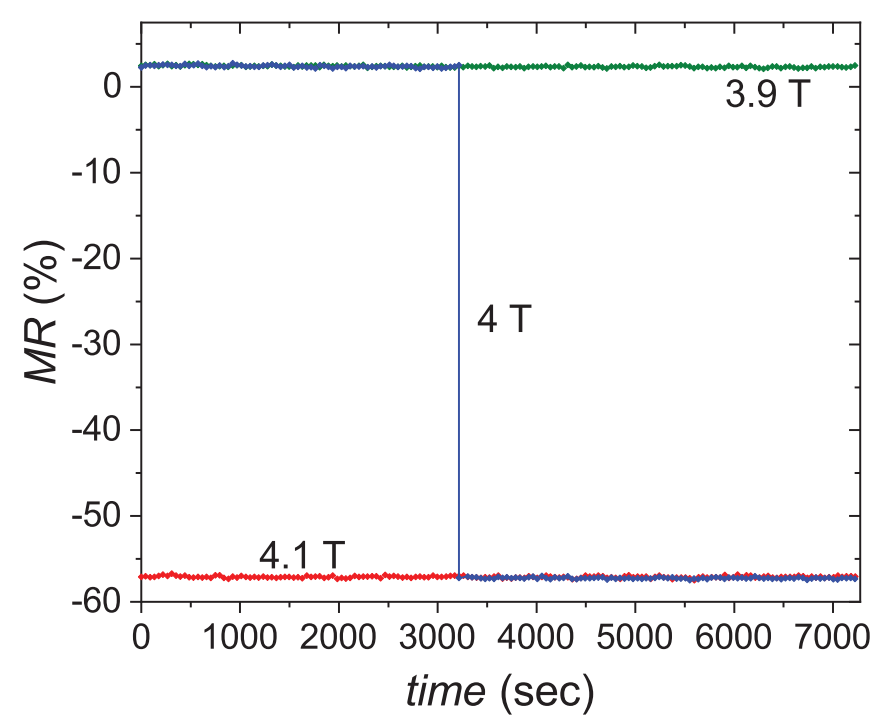

FIG. 10. Time dependence of the magnetoresistance recorded at the indicated applied fields for $\mathrm{La}_{0.85} \mathrm{Ce}_{0.15} \mathrm{Fe}_{12} \mathrm{~B}_{6}$ at $2.5 \mathrm{~K}$.

dependence measurements, the sample was cooled from 300 to $2.5 \mathrm{~K}$ in the absence of applied magnetic field. After the measurement temperature of $2.5 \mathrm{~K}$ is stabilized, a magnetic field is applied, and then the electrical resistivity is recorded vs time (duration of $7200 \mathrm{~s}$ ). The same experimental protocol was repeated several times applying various fields in steps of $0.1 \mathrm{~T}$, and the results are shown in Fig. 10. For an applied magnetic field of $4 \mathrm{~T}$, the curve displays giant resistive relaxation effects. The MR decreases abruptly from 2.5 to $-57 \%$, owing to a sudden formation of FM domains at the expense of the AFM phase. One can also emphasize that the spectacular and ultrasharp resistivity step occurs over a period smaller than the time interval separating two consecutive measurement points, i.e., $<40 \mathrm{~s}$ (which is required for averaging). The most salient feature in the present data is the huge spontaneous jump in electrical resistivity at a well-defined time when both external parameters (magnetic field and temperature) are kept constant. The transition time (incubation time) is found to be $\sim 3260 \mathrm{~s}$ in $\mu_{0} H=4 \mathrm{~T}$. Remarkably, this exceptional resistive relaxation effect found in the $\mathrm{La}_{0.85} \mathrm{Ce}_{0.15} \mathrm{Fe}_{12} \mathrm{~B}_{6}$ intermetallic compound exhibits a similarity to the peculiar behavior seen in standard martensitic transitions. The time dependence of the electrical resistivity for the Fe-31.7 at. \% Ni alloy [35] strikingly resembles Fig. 10, i.e., a sudden step is detected after an incubation time of $1020 \mathrm{~s}$.

Although the spontaneous step is seen on both resistive and magnetic relaxation data for the $\mathrm{La}_{0.85} \mathrm{Ce}_{0.15} \mathrm{Fe}_{12} \mathrm{~B}_{6}$ system, nevertheless, there is a discrepancy in the incubation time. The transition time differs from 3260 to $3810 \mathrm{~s}$ for resistive and magnetic [15] isothermal holding, respectively. This clearly demonstrates that the characteristic time associated with the sharp discontinuity is not a material constant. The phenomenal relaxation observed at $4 \mathrm{~T}$ is reminiscent of an explosive instability where the resistivity of the system endures a colossal change in a very short time interval [36]. The consistence seen in the magnetotransport and magnetic properties obviously reveals the strong coupling between charge and spin degrees of freedom in the $\mathrm{La}_{0.85} \mathrm{Ce}_{0.15} \mathrm{Fe}_{12} \mathrm{~B}_{6}$ compound.

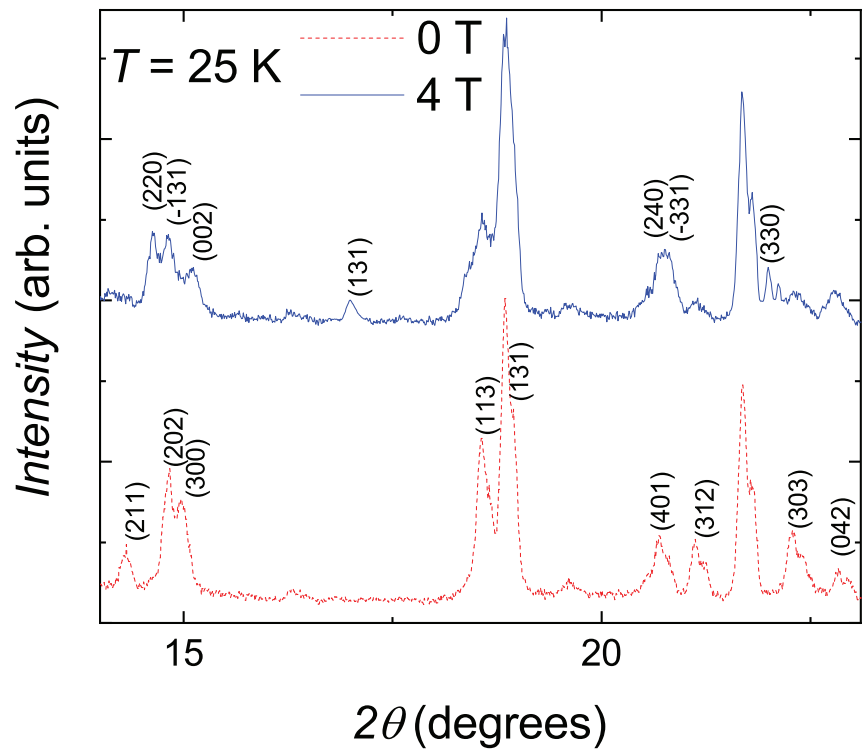

FIG. 11. Diffraction patterns measured at $25 \mathrm{~K}$ in magnetic fields of 0 and $4 \mathrm{~T}$ for the $\mathrm{La}_{0.85} \mathrm{Ce}_{0.15} \mathrm{Fe}_{12} \mathrm{~B}_{6}$ compound. Only the low angle part (from $14^{\circ}$ to $23^{\circ}$ ) of the diffraction pattern is shown to better illustrate development of phases with applied magnetic field.

\section{X-ray powder diffraction in applied magnetic fields}

The observation of anomalous features and multiple magnetic transitions by resistivity and magnetization characterizations urged us to carry out $\mathrm{x}$-ray powder diffraction investigations to establish the crystallographic structures of $\mathrm{La}_{0.85} \mathrm{Ce}_{0.15} \mathrm{Fe}_{12} \mathrm{~B}_{6}$ under various duplicated magnetic field and temperature conditions. Along with zero-field experiments, x-ray diffraction spectra were measured in a constant applied magnetic field at temperatures ranging from 15 and $100 \mathrm{~K}$. The diffractograms recorded at $25 \mathrm{~K}$ in applied fields of 0 and $4 \mathrm{~T}$ are displayed in Fig. 11. For the sake of clarity, only the region from $14^{\circ}$ to $23^{\circ} 2 \theta$ is depicted. Inspecting Fig. 11, one can immediately observe big differences between the diffraction profiles at zero magnetic field and under applied field. In $4 \mathrm{~T}$ external field, the x-ray powder diffraction pattern is significantly modified with the appearance of new Bragg peaks, revealing the presence of a magnetic-fieldinduced structural transition. At $25 \mathrm{~K}$ and $4 \mathrm{~T}$, two structurally distinct $\mathrm{La}_{0.85} \mathrm{Ce}_{0.15} \mathrm{Fe}_{12} \mathrm{~B}_{6}$ phases coexist, which is consistent with the first-order character of the transformation.

The Rietveld refinement of the complete diffractogram measured at $25 \mathrm{~K}$ in $4 \mathrm{~T}$ magnetic field is illustrated in Fig. 12. At $0 \mathrm{~T}$ (see Fig. 1) in the AFM ground state, as previously mentioned, the diffraction pattern of the compound reflects the pure rhombohedral structure adopting the $R \overline{3} m$ symmetry group (from now on called the Rhom phase). The additional Bragg reflections seen in the $4 \mathrm{~T} x$-ray diagram can be indexed in a monoclinic lattice with the $C 2 / \mathrm{m}$ space group (Mono phase). A good structure refinement was achieved in the $C 2 / \mathrm{m}$ symmetry group. According to the International Tables for Crystallography, all of the listed maximal nonisomorphic subgroups for the $R \overline{3} m$ crystal symmetry group are trigonal except $C 2 / m$. This monoclinic space group symmetry fits very well the high field diffraction data and is employed to describe the 


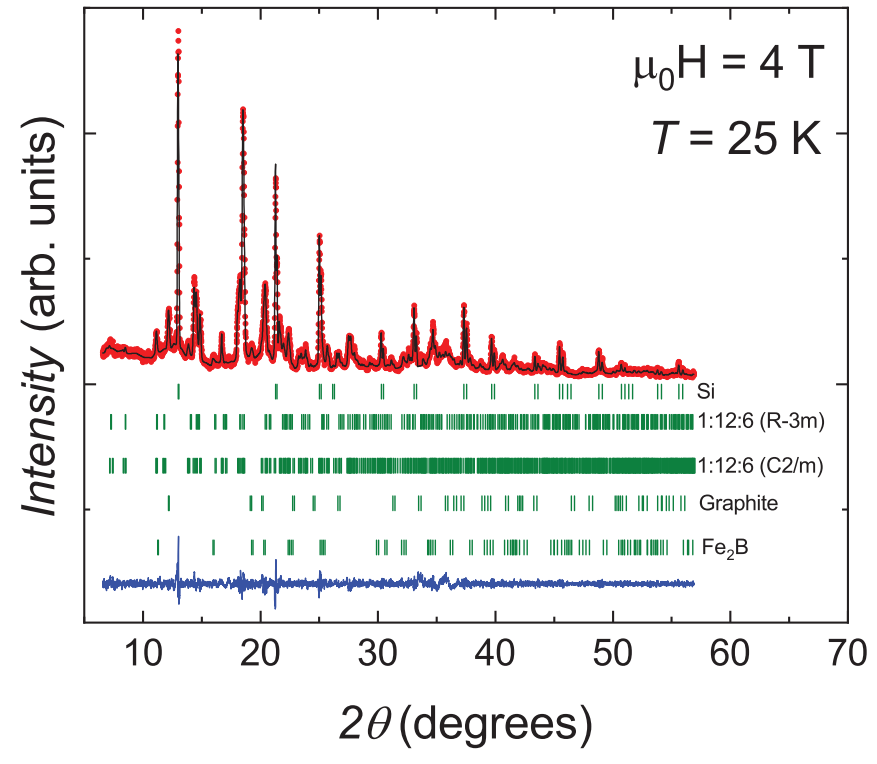

FIG. 12. Rietveld refinement of the x-ray powder diffraction pattern collected at $25 \mathrm{~K}$ in magnetic field of $\mu_{0} H=4 \mathrm{~T}$ for the $\mathrm{La}_{0.85} \mathrm{Ce}_{0.15} \mathrm{Fe}_{12} \mathrm{~B}_{6}$ compound. The observed (red dots) and calculated (black lines) patterns are shown. The blue line represents the difference $I_{\mathrm{obs}}-I_{\text {calc }}$. The vertical bars (olive) indicated positions of Bragg peaks for the different phases.

observed structural distortion. Consequently, it is likely to be the correct high magnetic field crystallographic structure of the $\mathrm{La}_{0.85} \mathrm{Ce}_{0.15} \mathrm{Fe}_{12} \mathrm{~B}_{6}$ intermetallic compound. Such a fieldinduced lattice distortion lowers the symmetry of the unit cell from trigonal to monoclinic, i.e., a spontaneous loss of the threefold symmetry in the $a b$ basal plane. The x-ray powder diffraction analyses reveal a change of the lattice symmetry across the magnetic-field-induced first-order AFM-FM transformation, in other words, a magnetostructural or coupled crystallographic-magnetic transition from a Rhom (AFM) to a Mono (FM) phase. The structural parameters and reliability factors derived from the fits at $25 \mathrm{~K}$ in 0 and $4 \mathrm{~T}$ are given in Table I. Temperature-dependent $\mathrm{x}$-ray powder diffraction measurements were also undertaken at $2 \mathrm{~T}$ between 15 and $100 \mathrm{~K}$; the obtained results were consistent with those at $4 \mathrm{~T}$.

Let us briefly describe the relationship between the monoclinic and rhombohedral lattices. The crystal lattice vectors of the two settings are connected according to $\mathbf{a}_{\text {Mono }}=-\frac{1}{3} \mathbf{a}_{\text {Rhom }}+\frac{1}{3} \mathbf{b}_{\text {Rhom }}-\frac{2}{3} \mathbf{c}_{\text {Rhom }} ; \quad \mathbf{b}_{\text {Mono }}=\mathbf{a}_{\text {Rhom }}+$ $\mathbf{b}_{\text {Rhom }} ; \mathbf{c}_{\text {Mono }}=\frac{1}{3} \mathbf{a}_{\text {Rhom }}-\frac{1}{3} \mathbf{b}_{\text {Rhom }}-\frac{1}{3} \mathbf{c}_{\text {Rhom }} ;$ so that $b_{\text {Mono }}=$ $a_{\text {Rhom }}$, and the rhombohedral crystallographic structure is described in the hexagonal triple cell. In the monoclinic crystal structure (space group $C 2 / m$ ), La/Ce atoms occupy one single Wyckoff position (2c), the $\mathrm{Fe}$ atoms are located on four inequivalent crystal sites $(\mathrm{Fe} 1 A$ in $4 i, \mathrm{Fe} 1 B$ in $8 j, \mathrm{Fe} 2 A$ in $8 j$, and $\mathrm{Fe} 2 B$ in $4 g$ ), and $\mathrm{B}$ atoms reside on two inequivalent positions (B1 in $4 i$ and $\mathrm{B} 2$ in $8 j$ ). The monoclinic unit cell contains 2 f.u., and altogether it is composed of 38 atoms (24 Fe, $12 \mathrm{~B}$, and $2 \mathrm{La} / \mathrm{Ce}$ ). The relationship between the two crystallographic arrangements is that each of the three 18 -fold positions from the rhombohedral crystal structure, which are initially occupied by $18 \mathrm{~B}$ and $2 \times 18 \mathrm{Fe}$, are split into pairs of independent eightfold and fourfold positions

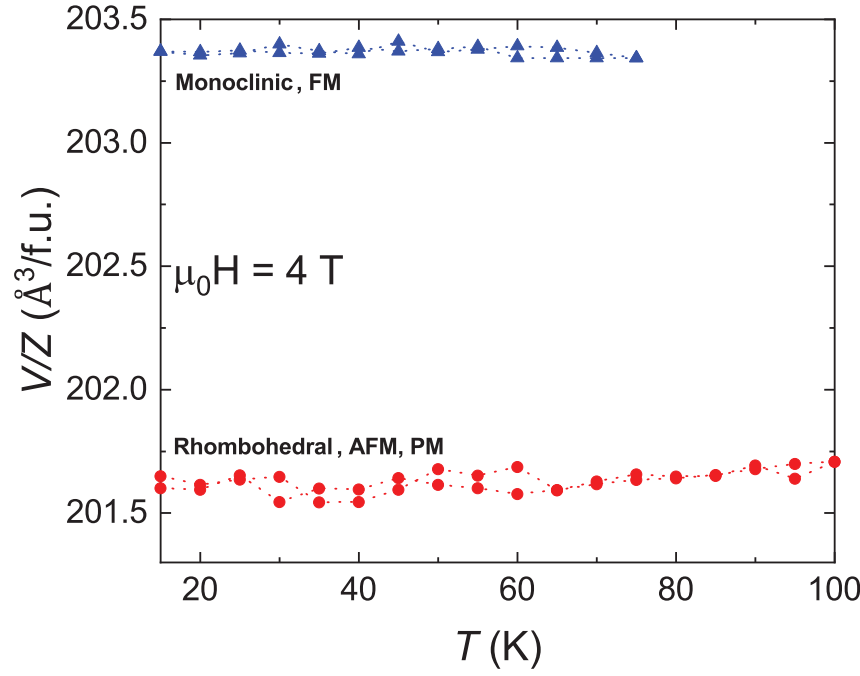

FIG. 13. Temperature dependence of the cell volume per formula unit for $\mathrm{La}_{0.85} \mathrm{Ce}_{0.15} \mathrm{Fe}_{12} \mathrm{~B}_{6}$ (both rhombohedral and monoclinic phases) derived from $\mathrm{x}$-ray diffraction measurements during heating and cooling in $\mu_{0} H=4 \mathrm{~T}$ applied magnetic field.

in the monoclinic atomic arrangement. The threefold site in the rhombohedral crystal system yields a twofold site in the monoclinic lattice.

The relationship between the rhombohedral (in hexagonal setting) and monoclinic cell volumes is as follows: $V_{\text {Mono }}$ $(C 2 / m)=\frac{2}{3} V_{\text {Rhom }}(R \overline{3} m)$. To directly compare the volumes of the two different atomic arrangements, we normalized their cell volumes to a chemical formula since $Z$ differs from one crystal structure to another. The thermal evolution of the normalized volumes in $4 \mathrm{~T}$ is reported in Fig. 13 including the data upon heating and cooling. In an applied magnetic field of $4 \mathrm{~T}$, the intermetallic compound $\mathrm{La}_{0.85} \mathrm{Ce}_{0.15} \mathrm{Fe}_{12} \mathrm{~B}_{6}$ presents an incomplete structural transition from a rhombohedral (AFM, PM) to a monoclinic (FM) lattice; both crystal structures coexist over a wide temperature range. At the lowest measurement temperature $(15 \mathrm{~K})$, the application of a $4 \mathrm{~T}$ magnetic field triggers $\sim 80 \%$ of the structural alteration, and the relative volume change associated with the field-driven magnetostructural transition amounts to $0.88 \%$. At $65 \mathrm{~K}$, the volume of the induced FM (Mono) phase is $0.89 \%$ larger than that of the PM (Rhom) one. The relative volume variations at the AFM-FM and FM-PM magnetic transitions are almost the same. The onset of the FM order in $\mathrm{La}_{0.85} \mathrm{Ce}_{0.15} \mathrm{Fe}_{12} \mathrm{~B}_{6}$ is characterized by a symmetry-lowering lattice distortion and a simultaneous volume expansion. Figure 14 presents the thermal dependence of the monoclinic structural parameters. The cell dimensions vary slowly at low temperatures and show larger changes in the vicinity of the transition temperature. One can observe an extremely anisotropic cell expansion: the lattice expands along the $b$ direction, while it contracts along the other two principal crystallographic $a$ and $c$ axes. Moreover, the lattice parameters $a, b$, and $c$, and the angle $\beta$ exhibit a large temperature hysteresis.

Figure 15 represents the evolution of the fractions of the monoclinic (upper panel) and rhombohedral (lower panel) phases with temperature upon warming and cooling in $\mu_{0} H=$ 

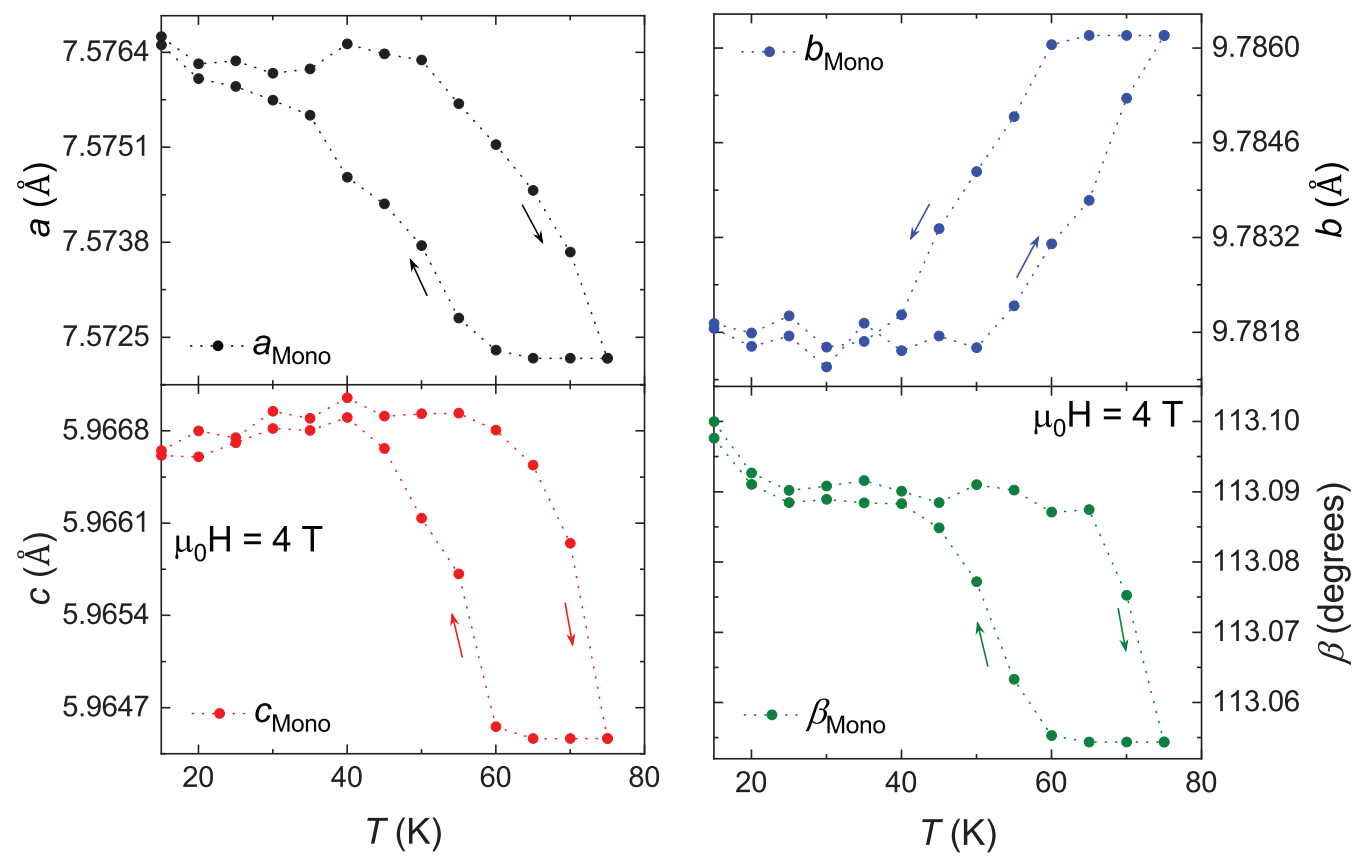

FIG. 14. Temperature dependence of the lattice parameters $a, b$, and $c$, and Bragg angle $\beta$ of the monoclinic phase determined from x-ray powder diffraction measurements during heating and cooling in $\mu_{0} H=4 \mathrm{~T}$ applied magnetic field.

2 and $4 \mathrm{~T}$. From these results, it is obvious that the ratio between the rhombohedral and monoclinic phases depends strongly on the strength of the applied magnetic field. A huge thermal hysteresis of $15 \mathrm{~K}$ exists between ZFC and FC measuring protocols in $4 \mathrm{~T}$. The behavior seen in Fig. 15 follows from a competition between the growth of the Mono (FM) phase as the temperature is lowered and the reduction of the Rhom (AFM, PM) proportion. The transition is incomplete upon cooling in $2 \mathrm{~T}$ with $40 \%$ of the rhombohedral $\mathrm{La}_{0.85} \mathrm{Ce}_{0.15} \mathrm{Fe}_{12} \mathrm{~B}_{6}$ transformed into the monoclinic polymorph at $15 \mathrm{~K}$. In $2 \mathrm{~T}$ applied magnetic field, the rhombohedral allotrope remains the majority phase over the investigated temperature interval. The sample gets transformed partially into the monoclinic phase, which becomes dominant $(\sim 80 \%)$ in $4 \mathrm{~T}$ at $15 \mathrm{~K}$. When heated and cooled in external fields of 2 and $4 \mathrm{~T}, \mathrm{La}_{0.85} \mathrm{Ce}_{0.15} \mathrm{Fe}_{12} \mathrm{~B}_{6}$ presents crystallographic inhomogeneity or structurally heterogeneous state (coexistence of polymorphs) below the Curie point. Above this temperature, the system adopts rhombohedral structure and recovers homogeneity. Similar structurally and magnetically inhomogeneous states were also observed in some intermetallic systems like $\mathrm{Gd}_{5}\left(\mathrm{Si}_{x} \mathrm{Ge}_{1-x}\right)_{4}[9,10,37,38]$, Si-doped $\mathrm{CeFe}_{2}$ [39], MnAs [1,40], and generally found in the colossal magnetoresistive manganese-based perovskites, where they are often called phase-segregated states $[41,42]$.

The weighted average cell volume in 2 and $4 \mathrm{~T}$ is plotted against temperature in Fig. 16. The mean volume displays a transition accompanied by a temperature hysteresis which is consistent with a first-order phase transition. The reduction (growth) of the content of the monoclinic polymorph and the growth (reduction) of the concentration of the rhombohedral phase during heating (cooling) leads to a reduction (increase) of the average volume. For an applied magnetic field of $4 \mathrm{~T}$, the weighted mean cell volume presents a negative thermal expansion (NTE) phenomenon over the temperature interval between 35 and $80 \mathrm{~K}$ with an average volumetric thermal expansion coefficient $\alpha_{V}=-195 \times 10^{-6} \mathrm{~K}^{-1}$. This huge NTE effect originates from the strong magnetolattice coupling. It is worth noting that our experimental volumetric NTE coefficient compares well with the giant negative thermal expansion reported in $(\mathrm{Hf}, \mathrm{Ta}) \mathrm{Fe}_{2}$ itinerant-electron metamagnets $\left(\alpha_{V}=\right.$ $\left.-164 \times 10^{-6} \mathrm{~K}\right)[43,44]$ and is on the same order of magnitude as the colossal NTE observed in $\operatorname{Mn}_{0.98} \mathrm{CoGe}\left(\alpha_{V}=\right.$ $\left.-423 \times 10^{-6} \mathrm{~K}^{-1}\right)[45]$ and $\mathrm{Bi}_{0.95} \mathrm{La}_{0.05} \mathrm{NiO}_{3}\left(\alpha_{V}=-413 \times\right.$ $\left.10^{-6} \mathrm{~K}^{-1}\right)$ [46].

The thermomagnetic behavior of Fig. 1 may be directly correlated with the phase fractions derived from Rietveld refinements of the x-ray powder diffraction patterns by comparing the normalized magnetization data with the concentration of the monoclinic $\mathrm{La}_{0.85} \mathrm{Ce}_{0.15} \mathrm{Fe}_{12} \mathrm{~B}_{6}$. The compilation of the results of $\mathrm{x}$-ray diffraction studies and magnetic measurements is presented in Fig. 17. The change of the monoclinic phase content with temperature agrees well with the percentage of the FM phase obtained from magnetization in the same applied fields and confirms the intimate coupling between magnetic ordering and the crystal structure in this intermetallic compound. However, there are small discrepancies in values between the magnetic and $\mathrm{x}$-ray powder diffraction data, which we attribute to arise from the following reasons: (i) the magnetization normalization yields some errors due to incompleteness of the transformation and (ii) the intrinsic difference between the magnetization and $\mathrm{x}$-ray diffraction experiments. In the magnetization measurement, a recorded signal reflects the alignment of magnetic moments and magnetic domains, as well as a structural change, while $\mathrm{x}$-ray powder diffraction is only sensitive to a structural alteration. Furthermore, the PM and AFM states have nonzero magnetizations in nonzero external magnetic fields. 


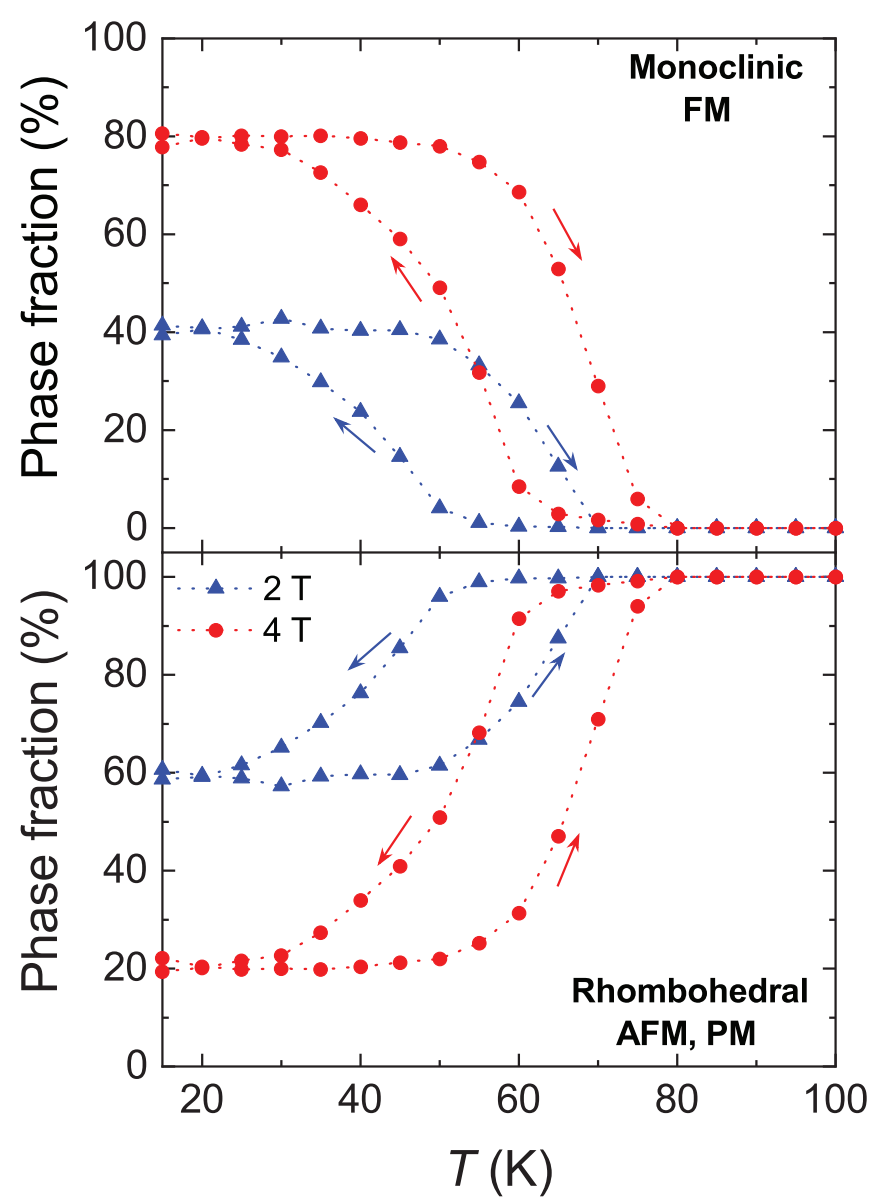

FIG. 15. Temperature dependence of the phase fractions of both rhombohedral and monoclinic crystal structures of $\mathrm{La}_{0.85} \mathrm{Ce}_{0.15} \mathrm{Fe}_{12} \mathrm{~B}_{6}$ as determined from $\mathrm{x}$-ray powder diffraction measurements during heating and cooling in $\mu_{0} H=2$ and $4 \mathrm{~T}$ applied magnetic fields.

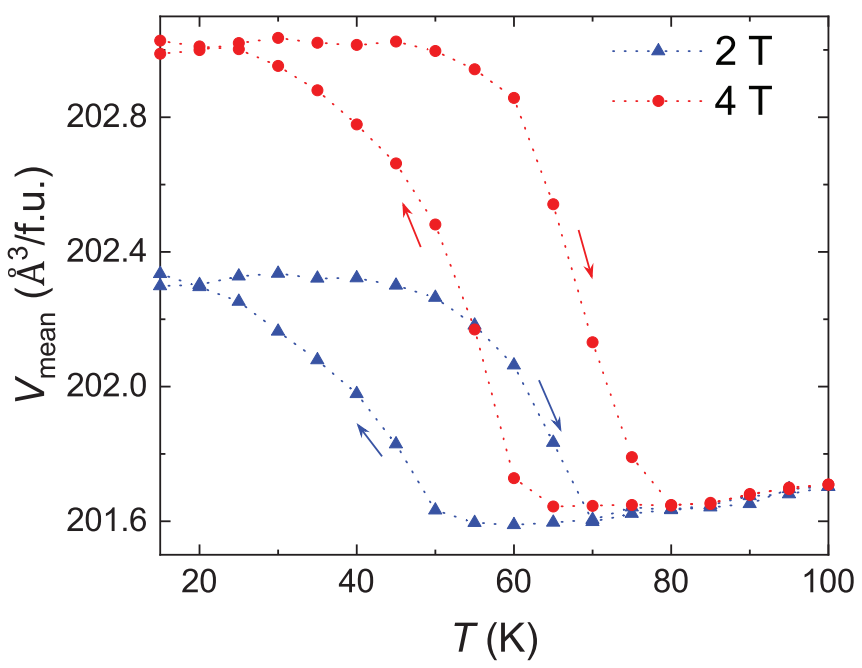

FIG. 16. Temperature dependence of the mean cell volume per formula unit determined from x-ray powder diffraction measurements during heating and cooling in $\mu_{0} H=2$ and $4 \mathrm{~T}$ applied magnetic fields.

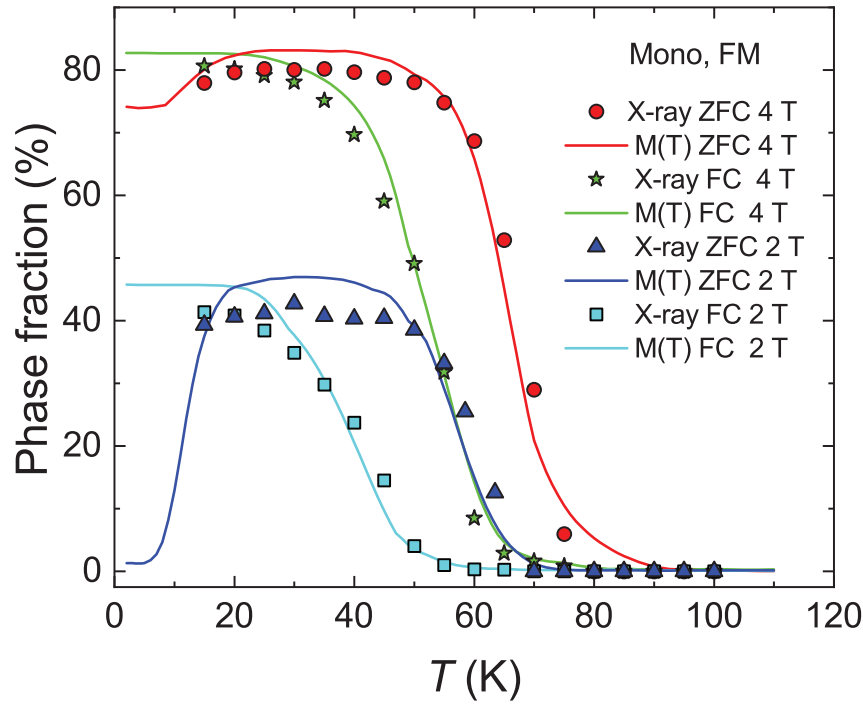

FIG. 17. Temperature dependence of the concentration of the monoclinic phase of $\mathrm{La}_{0.85} \mathrm{Ce}_{0.15} \mathrm{Fe}_{12} \mathrm{~B}_{6}$ as determined from $\mathrm{x}$-ray powder diffraction (symbols) and magnetization (lines) measurements during heating and cooling in $\mu_{0} H=2$ and $4 \mathrm{~T}$ fields.

To further elucidate the lattice response at the metamagnetic transition seen in both magnetization and MR isotherms and as well to get a deeper insight into the correlations between the structural and magnetic properties of $\mathrm{La}_{0.85} \mathrm{Ce}_{0.15} \mathrm{Fe}_{12} \mathrm{~B}_{6}$, the magnetic field dependence of the crystal structure was examined at a fixed temperature of $25 \mathrm{~K}$ (x-ray isotherm). For this experiment, the sample was thermally demagnetized at room temperature and zero-field cooled down to $25 \mathrm{~K}$. Thereafter, the field was increased between 0 and the maximum attainable value of $5.5 \mathrm{~T}$ at regular steps and diffraction patterns measured at every magnetic field step. The diffraction peaks, which correspond to the monoclinic crystal structure, emerge at $\mu_{0} H \geqslant 0.5 \mathrm{~T}$. When the applied field increases, the intensities of the Bragg reflections corresponding to the rhombohedral phase are reduced, while those of the Bragg peaks of the monoclinic polymorph are increased. In the magnetic field range between 0.5 and $5.5 \mathrm{~T}$, no other changes, except for the progressive reapportionment of intensities of the diffraction lines belonging to the two distinct $\mathrm{La}_{0.85} \mathrm{Ce}_{0.15} \mathrm{Fe}_{12} \mathrm{~B}_{6}$ polymorphs, are detected in the $\mathrm{x}$-ray spectra.

The magnetic field dependence of the normalized cell volumes at $25 \mathrm{~K}$ is presented in Fig. 18(a). The Rietveld analysis of the $\mathrm{x}$-ray isotherm indicates that the variations of the structural parameters of each allotrope during isothermal magnetization are very small; hence, the unit cell volumes of both phases are nearly field independent. However, the magnetostriction due to the crystallographic transition from Rhom to Mono is large. The cell volume of the Rhom phase is significantly smaller than that of the Mono phase. The maximum forced volume magnetostriction related to the Rhom (AFM)-Mono (FM) magnetostructural transformation is estimated to be $0.9 \%$ at $25 \mathrm{~K}$ and $5.5 \mathrm{~T}$. The magnitude of the volume change in $\mathrm{La}_{0.85} \mathrm{Ce}_{0.15} \mathrm{Fe}_{12} \mathrm{~B}_{6}$ compares well with the isotropic forced volume magnetostriction across the magnetic-field-induced metamagnetic transition 

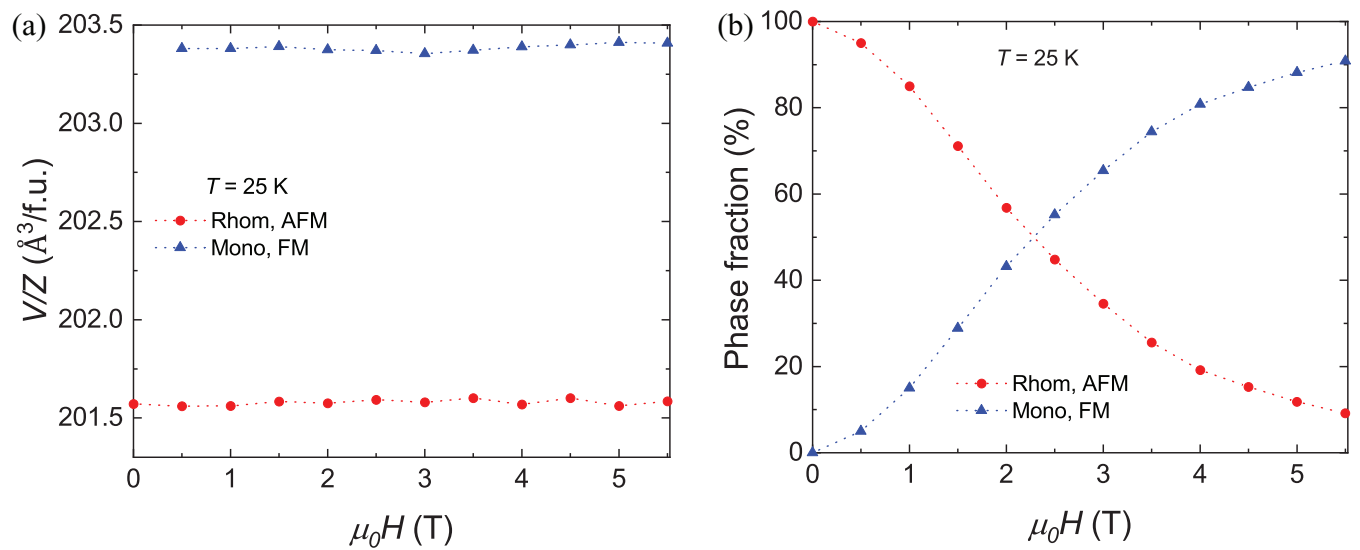

FIG. 18. Magnetic field dependence of the (a) cell volume per chemical formula for $\mathrm{La}_{0.85} \mathrm{Ce}_{0.15} \mathrm{Fe}_{12} \mathrm{~B}_{6}$ and (b) phase fractions of both rhombohedral and monoclinic crystal structures determined from $\mathrm{x}$-ray powder diffraction measurements at $25 \mathrm{~K}$.

in iron-rich intermetallic systems such as $\mathrm{La}\left(\mathrm{Fe}_{x} \mathrm{Al}_{1-x}\right)_{13}$ [47] and $\mathrm{La}\left(\mathrm{Fe}_{x} \mathrm{Si}_{1-x}\right)_{13}$ [48]. Indeed, a relative volume variation as large as $1 \%$ was observed in the pseudobinary $\mathrm{La}\left(\mathrm{Fe}_{0.87} \mathrm{Al}_{0.13}\right)_{13}$ alloy [47]. Volume magnetostrictions of 0.9 and $1.5 \%$ were reported for $\mathrm{La}\left(\mathrm{Fe}_{0.86} \mathrm{Si}_{0.14}\right)_{13}$ and $\mathrm{La}\left(\mathrm{Fe}_{0.88} \mathrm{Si}_{0.12}\right)_{13}$, respectively [48]. Figure 18(b) illustrates the field evolution of the proportion of each phase at $25 \mathrm{~K}$, and the corresponding weighted mean cell volume is plotted in Fig. 19. Upon increasing applied magnetic field, the amount of the Mono (FM) component gradually grows with the simultaneous decrease of the Rhom (AFM) phase. At a field of $\approx 2.25 \mathrm{~T}$, the volume fraction of the two polymorphs is close to $1: 1$ ratio, a structurally and magnetically heterogeneous state. At the highest applied magnetic field of $5.5 \mathrm{~T}, 91 \%$ of the rhombohedral structure is converted to the Mono phase. At $25 \mathrm{~K}$, the average volume follows the changes of the concentration of the Mono phase. In the present compound, the magnetic field promotes the development of the high-volume FM phase.

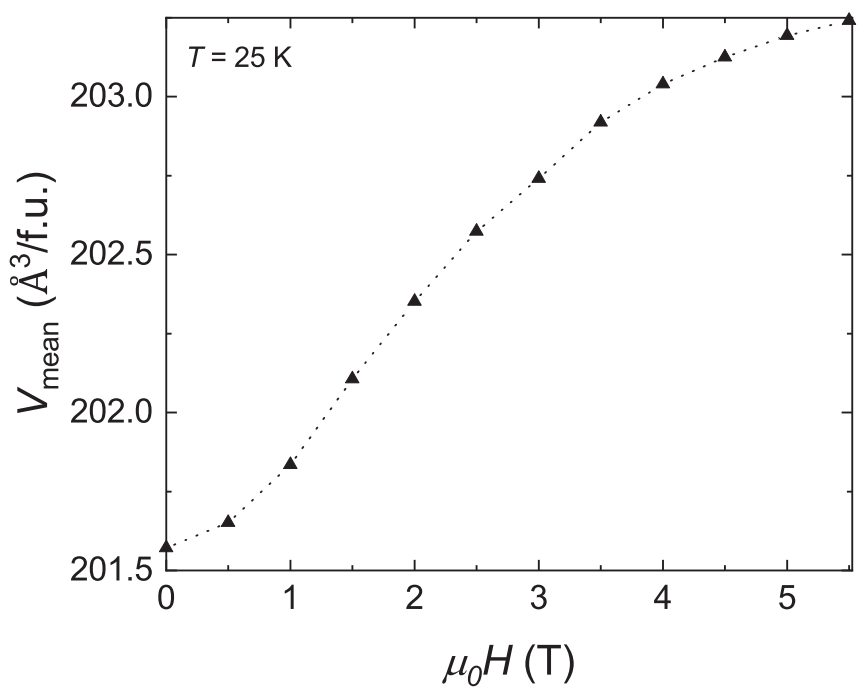

FIG. 19. Magnetic field dependence of the mean cell volume per chemical formula determined from powder diffraction measurements at $25 \mathrm{~K}$ for $\mathrm{La}_{0.85} \mathrm{Ce}_{0.15} \mathrm{Fe}_{12} \mathrm{~B}_{6}$.
The relative variation of the weighted mean volume is presented in Fig. 20 together with the isothermal magnetization data collected at $25 \mathrm{~K}$. Both curves show nearly the same behavior: the average cell volume increases quickly as the magnetization undergoes a magnetic transition. The metamagnetic process is unmistakably visible in the relative change of the mean volume. These data demonstrate that the metamagnetic transition is accompanied by large magnetovolume effects and shed light on the strong coupling between the crystal and magnetic lattices. The magnetic field stimulates a transformation from a low-volume, low-magnetization to a high-volume, high-magnetization phase.

\section{E. Discussion}

The experimental results presented in Secs. III B and III C reveal that the magnetic and magnetotransport behaviors of $\mathrm{La}_{0.85} \mathrm{Ce}_{0.15} \mathrm{Fe}_{12} \mathrm{~B}_{6}$ are most interesting at very low temperature where the magnetization and MR isotherms display steep jumps. It is essential at this juncture to consider the issue of the origin of these sharp discontinuities. It is quite evident that

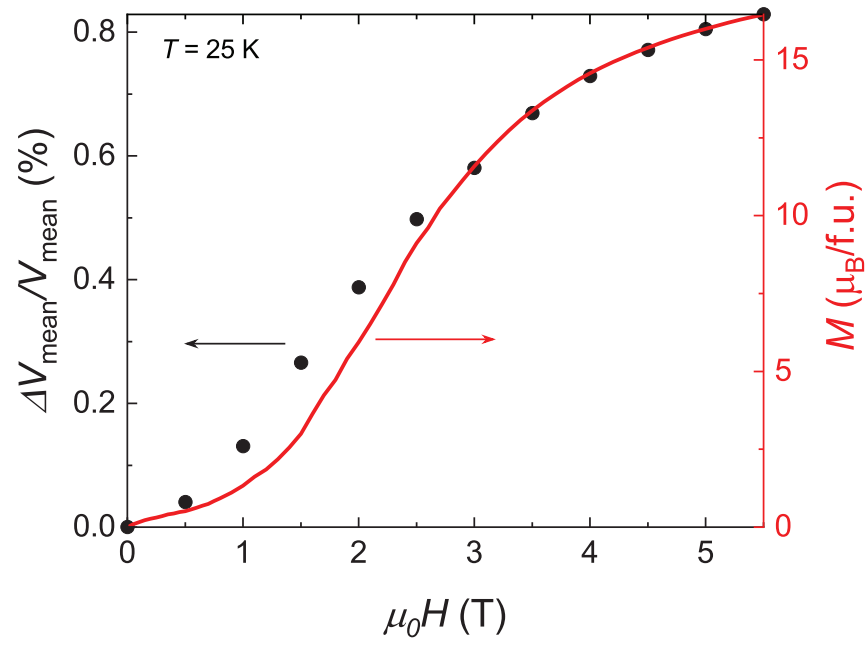

FIG. 20. Magnetic field dependence of the relative volume change and the magnetization at $25 \mathrm{~K}$ of $\mathrm{La}_{0.85} \mathrm{Ce}_{0.15} \mathrm{Fe}_{12} \mathrm{~B}_{6}$. 
the steplike transition phenomena observed in the itinerantelectron system $\mathrm{La}_{0.85} \mathrm{Ce}_{0.15} \mathrm{Fe}_{12} \mathrm{~B}_{6}$ are not purely magnetic in origin, yet they have a contribution from the strong magnetoelastic coupling. Even though several explanations have been suggested for these unusual and anomalous features in oxides and intermetallics, the most plausible one appears to be a scenario based on the martensiticlike effect stimulated by external magnetic field [49-51].

Let us depict the occurrence of avalanchelike metamagnetic transitions in the magnetization and MR curves within the framework of such a martensiticlike process. Here, $\mathrm{La}_{0.85} \mathrm{Ce}_{0.15} \mathrm{Fe}_{12} \mathrm{~B}_{6}$ exhibits an AFM ground state with a rhombohedral crystal structure. However, with the application of a suitable magnetic field, the FM phase (monoclinic cell) develops as a function of temperature, field, as well as time inside the AFM matrix. The magnetic-field-driven lattice distortion (martensiticlike transition) between the rhombohedral and monoclinic crystallographic structures generates elastic strains at the AFM/FM interfaces. Upon applying magnetic field, FM regions are likely to grow, but the interfacial (martensitic) constraints act against this to block the development of the FM domains. Since the external applied field is gradually increased, the driving force acting on the magnetic moments raises as well. When the magnetic force is strong enough to overcome the elastic strain energy, the FM component evolves in a catastrophic manner, leading to an abrupt change of magnetization and resistivity. Consequently, the overall transition proceeds by successive sudden jumps separated by plateaus. These ultrasharp steps seen in the resistivity and magnetization data can be considered as a burstlike growth of the FM component within the AFM matrix. The field-induced structural distortion which is driven by magnetoelastic coupling, is likely responsi- ble for the stepwise transitions in the $\mathrm{La}_{0.85} \mathrm{Ce}_{0.15} \mathrm{Fe}_{12} \mathrm{~B}_{6}$ alloy.

\section{CONCLUDING REMARKS}

We have performed a detailed study of the structural, magnetic, and transport properties of $\mathrm{La}_{0.85} \mathrm{Ce}_{0.15} \mathrm{Fe}_{12} \mathrm{~B}_{6}$ as a function of temperature, magnetic field, and time. Multiple steplike metamagnetic transitions were observed at low temperatures. X-ray powder diffraction indicated that the first-order AFM-FM and PM-FM phase transformations are associated with a symmetry-lowering rhombohedralto-monoclinic structural transition. The magnetic state is intimately related to its crystallography: the AFM and PM states adopt the rhombohedral structure $(R \overline{3} \mathrm{~m})$ but the monoclinic symmetry $(\mathrm{C} 2 / \mathrm{m})$ is associated with the FM order. This simultaneous magnetic-crystallographic transformation is accompanied by large magnetoelastic and giant negative MR effects. A peculiarly anisotropic thermal expansion and giant NTE effect with a volumetric thermal expansion coefficient $\alpha_{V}=-195 \times 10^{-6} \mathrm{~K}^{-1}$ were found. At constant applied magnetic field and temperature, a colossal spontaneous MR jump occurs after a very long incubation time of $\sim 3260 \mathrm{~s}$.

Magnetic-field-dependent neutron powder diffraction experiments are planned in the near future to establish the magnetic structure of the FM (monoclinic) phase and to gain a deeper understanding of the coupling between the magnetic and crystal lattices in this itinerant-electron metamagnetic compound. The field-induced crystallographic transition reported in this paper may turn out to be key in deciphering future electronic band structure calculations and theoretical studies of the physical properties of this intriguing system.
[1] J. Mira, F. Rivadulla, J. Rivas, A. Fondado, T. Guidi, R. Caciuffo, F. Carsughi, P. G. Radaelli, and J. B. Goodenough, Phys. Rev. Lett. 90, 097203 (2003).

[2] A. Zieba, Y. Shapira, and S. Foner, Phys. Lett. A 91, 243 (1982).

[3] C. P. Bean and D. S. Rodbell, Phys. Rev. 126, 104 (1962).

[4] A. Asamitsu, Y. Moritomo, Y. Tomioka, T. Arima, and Y. Tokura, Nature (London) 373, 407 (1995).

[5] E. M. Levin, V. K. Pecharsky, K. A. Gschneidner, Jr., and G. J. Miller, Phys. Rev. B 64, 235103 (2001).

[6] C. Magen, L. Morellon, P. A. Algarabel, C. Marquina, and M. R. Ibarra, J. Phys.:Condens. Matter 15, 2389 (2003).

[7] S. B. Roy, M. K. Chattopadhyay, P. Chaddah, J. D. Moore, G. K. Perkins, L. F. Cohen, K. A. Gschneidner, Jr., and V. K. Pecharsky, Phys. Rev. B 74, 012403 (2006).

[8] V. K. Pecharsky, A. P. Holm, K. A. Gschneidner, Jr., and R. Rink, Phys. Rev. Lett. 91, 197204 (2003).

[9] V. K. Pecharsky and K. A. Gschneidner, Jr., Adv. Mater. 13, 683 (2001).

[10] L. Morellon, P. A. Algarabel, M. R. Ibarra, J. Blasco, B. GarcíaLanda, Z. Arnold, and F. Albertini, Phys. Rev. B 58, R14721(R) (1998).

[11] V. K. Pecharsky, and K. A. Gschneidner, Jr., Phys. Rev. Lett. 78, 4494 (1997).
[12] L. V. B. Diop, O. Isnard, and J. Rodríguez-Carvajal, Phys. Rev. B 93, 014440 (2016).

[13] S. Fujieda, K. Fukamichi, and S. Suzuki, J. Magn. Magn. Mater 421, 403 (2017).

[14] L. V. B. Diop and O. Isnard, Appl. Phys. Lett. 108, 132401 (2016).

[15] L. V. B. Diop and O. Isnard, Phys. Rev. B 97, 014436 (2018).

[16] L. V. B. Diop and O. Isnard, J. Appl. Phys. 119, 213904 (2016).

[17] L. V. B. Diop, O. Isnard, Z. Arnold, J. P. Itié, J. Kastil, and J. Kamarad, Solid State Comm. 252, 29 (2017).

[18] G. I. Miletic and Z. Blazina, J. Magn. Magn. Mater. 323, 2340 (2011).

[19] G. I. Miletic and Z. Blazina, J. Alloys Compd. 430, 9 (2007).

[20] M. Rosenberg, T. Sinnemann, M. Mittag, and K. H. J. Buschow, J. Alloys Compd. 182, 145 (1992).

[21] Q. A. Li, C. H. de Groot, F. R. de Boer, and K. H. J. Buschow, J. Alloys Compd. 256, 82 (1997).

[22] M. Mittag, M. Rosenberg, and K. H. J. Buschow, J. Magn. Magn. Mater. 82, 109 (1989).

[23] K. H. J. Buschow, D. B. de Mooij, and H. M. van Noort, J. Less-Common Met. 125, 135 (1986). 
[24] F. Mesquita, S. G. Magalhaes, P. Pureur, L. V. B. Diop, and O. Isnard, Phys. Rev. B 101, 224414 (2020).

[25] A. Barlet, J. C. Genna, and P. Lethuillier, Cryogenic 31, 801 (1991).

[26] T. Faske and W. Donner, J. Appl. Crystallogr. 51, 761 (2018).

[27] J. Rodríguez-Carvajal, Physica B 192, 55 (1993).

[28] K. Niihara and S. Yajima, Chem. Lett. 1, 875 (1972).

[29] Yu. B.Kuz'ma, G. V. Chernyak, and N. F. Chaban, Dopov. Akad. Nauk. Ukr. RSR Ser. A 12, 80 (1981).

[30] W. Jung and D. Quentmeier, Z. Kristallogr. 151, 121 (1980).

[31] E. M. Levin, K. A. Gschneidner, Jr., and V. K. Pecharsky, Phys. Rev. B 65, 214427 (2002).

[32] T. Moriya, Spin Fluctuations in Itinerant Electron Magnetism (Springer-Verlag, Berlin, 1985).

[33] H. Yamada and T. Goto, Phys. Rev. B 68, 184417 (2003).

[34] T. Goto, K. Fukamichi, and H. Yamada, Physica B 300, 167 (2001).

[35] T. Kakeshita, J. Katsuyama, T. Fukuda, and T. Saburi, Mater. Sci. Eng. A 312, 219 (2001).

[36] V. Hardy, A. Maignan, S. Hebert, C. Yaicle, C. Martin, M. Hervieu, M. R. Lees, G. Rowlands, D. M. Paul, and B. Raveau, Phys. Rev. B 68, 220402(R) (2003).

[37] Ya. Mudryk, A. P. Holm, K. A. Gschneidner, Jr., and V. K. Pecharsky, Phys. Rev. B 72, 064442 (2005).

[38] Ya. Mudryk, D. Paudyal, V. K. Pecharsky, and K. A. Gschneidner, Jr., Phys. Rev. B 77, 024408 (2008).

[39] A. Haldar, N. K. Singh, Ya. Mudryk, K. G. Suresh, A. K. Nigam, and V. K. Pecharsky, Solid State Comm. 150, 879 (2010).

[40] F. Ishikawa, K. Koyama, K. Watanabe, and H. Wada, Jpn. J. Appl. Phys. 42, L918 (2003).
[41] A. I. Kurbakov, V. A. Ryzhov, V. V. Runov, E. O. Bykov, I. I. Larionov, V. V. Deriglazov, C. Martin, and A. Maignan, Phys. Rev. B 100, 184424 (2019).

[42] J. B. Goodenough, in Handbook on the Physics and Chemistry of Rare Earths, edited by K. A. Gschneidner, Jr., J. C. Bünzli, and V. K. Pecharsky, (Elsevier, New York, 2003), Vol.33, p. 249, Chap. 214.

[43] L. V. B. Diop, O. Isnard, M. Amara, F. Gay, and J. P. Itié, J. Alloys Compd. 845, 156310 (2020).

[44] B. Li, X. H. Luo, H. Wang, W. J. Ren, S. Yano, C. W. Wang, J. S. Gardner, K. D. Liss, P. Miao, S. H. Lee, T. Kamiyama, R. Q. Wu, Y. Kawakita, and Z. D. Zhang, Phys. Rev. B 93, 224405 (2016).

[45] J. Lin, P. Tong, K. Zhang, H. Tong, X. Guo, C. Yang, Y. Wu, M. Wang, S. Lin, L. Chen, W. Song, and Y. Sun, Appl. Phys. Lett. 109, 241903 (2016).

[46] M. Azuma, W. Chen, H. Seki, M. Czapski, S. Olga, K. Oka, M. Mizumaki, T. Watanuki, N. Ishimatsu, N. Kawamura, S. Ishiwata, M. G. Tucker, Y. Shimakawa, and J. P. Attfield, Nature Commun. 2, 347 (2011).

[47] K. Irisawa, A. Fujita, K. Fukamichi, M. Yamada, H. Mitamura, T. Goto, and K. Koyama, Phys. Rev. B 70, 214405 (2004).

[48] A. Fujita, Y. Akamatsu, and K. Fukamichi, J. Appl. Phys. 85, 4756 (1999).

[49] V. Hardy, S. Majumdar, S. J. Crowe, M. R. Lees, D. McK. Paul, L. Hervé, A. Maignan, S. Hébert, C. Martin, C. Yaicle, M. Hervieu, and B. Raveau, Phys. Rev. B 69, 020407(R) (2004).

[50] B. Maji, K. G. Suresh, and A. K. Nigam, Europhys. Lett. 91, 37007 (2010).

[51] A. Haldar, K. G. Suresh, and A. K. Nigam, Phys. Rev. B 78, 144429 (2008). 